\title{
OPTIMAL EXIT TIME FROM CASINO GAMBLING: STRATEGIES OF PRECOMMITTED AND NAIVE GAMBLERS*
}

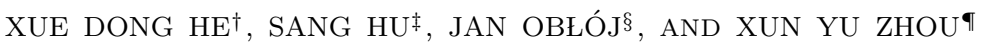

\begin{abstract}
We consider a casino gambling model with an indefinite end date and gamblers endowed with cumulative prospect theory preferences. We study the optimal strategies of a precommitted gambler, who commits her future selves to the strategy she sets up today, and of a naive gambler, who is unaware of time-inconsistency and may alter her strategy at any time. We identify conditions under which the precommitted gambler, asymptotically, adopts a loss-exit strategy, a gain-exit strategy, or a nonexit strategy. For a specific parameter setting when the utility function is piecewise power and the probability weighting functions are concave power, we derive the optimal strategy of the precommitted gambler in closed form whenever it exists, via solving an infinitedimensional program. Finally, we study the actual behavior of the naive gambler and highlight its marked differences from that of the precommitted gambler. In particular, for most empirically relevant cumulative prospect theory parameter values, a precommitted gambler takes a loss-exit strategy while a naive agent does not stop with probability one at any loss level.
\end{abstract}

Key words. casino gambling, cumulative prospect theory, optimal stopping, precommitted gamblers, naive gamblers, optimal strategies

AMS subject classifications. 91G80, 60G40, 91B16

DOI. $10.1137 / 18 \mathrm{M} 1174738$

1. Introduction. We analyze theoretically, and compare, the behavior of different types of gamblers in a casino gambling model, which is a generalization of the one initially proposed by Barberis in [5]. A gambler, consecutively playing out a fair bet in a casino, needs to determine the best time to stop playing and leave, with the objective to maximize her preference value of the final wealth, while the preference is dictated by the cumulative prospect theory (CPT) [33].

Barberis [5] proposes such a model for the first time with five periods and Markovian and nonrandomized strategies and derives the gambler's strategy by exhaustive search. The author finds that when the gambler uses the same CPT preferences to revisit the gambling problem in the future, e.g., at time $t>0$, the optimal gambling strategy solved at time 0 is typically no longer optimal. This time-inconsistency arises from the probability weighting in CPT. If the gambler is precommitted, she commits

*Received by the editors March 9, 2018; accepted for publication (in revised form) March 20, 2019; published electronically May 28, 2019. The main results in this paper are contained in the Ph.D. thesis of Sang Hu entitled "Optimal Exit Strategies of Behavioral Gamblers [21]," which was submitted to the Chinese University of Hong Kong (CUHK) in September 2014.

http://www.siam.org/journals/sicon/57-3/M117473.html

Funding: The first author acknowledges financial support from the General Research Fund of the Research Grants Council of Hong Kong SAR (project 14200917). The third author acknowledges support from ERC Starting Grant RobustFinMath 335421. The fourth author acknowledges financial support through a start-up grant at Columbia University and through the FDT Center for Intelligent Asset Management.

${ }^{\dagger}$ Room 609, William M.W. Mong Engineering Building, Department of Systems Engineering and Engineering Management, The Chinese University of Hong Kong, Shatin, N.T., Hong Kong (xdhe@se.cuhk.edu.hk).

${ }^{\ddagger}$ School of Science and Engineering, The Chinese University of Hong Kong, Shenzhen, China (husang@cuhk.edu.cn).

$\S$ Mathematical Institute, The Oxford-Man Institute of Quantitative Finance and St John's College, University of Oxford, Oxford, UK (Jan.Obloj@maths.ox.ac.uk).

IDepartment of Industrial Engineering and Operations Research, Columbia University, New York, NY 10027 (xz2574@columbia.edu). 
her future selves to the strategy solved at time 0. If she is not aware of the timeinconsistency, then the gambler is said to be naive. The actual strategy implemented by a naive gambler will often be dramatically different from the strategy planned at time 0 (which would be implemented by a precommitted gambler). For example, [5, Figure 4] shows that for a large range of parameter specifications the naive gambler entering the casino with a loss-exit plan ends up with a gain-exit strategy. Barberis [5] also studies the gambling behavior of so-called sophisticated gamblers without commitment, who know their future selves will not follow today's strategy and thus adjust today's strategy accordingly. The author finds that these gamblers never enter the casino. $^{1}$

He et al. [16] show that, for Barberis's model, external randomization such as tossing coins or path-dependent (namely, non-Markovian) strategies can strictly improve the gambler's CPT preference value. Moreover, the latter mechanism is equivalent to the former in the sense of reaching the same objective value; hence there is no need to consider path-dependence if randomization is in place. This finding is consistent with the use of random devices by individuals in various contexts; see, for instance, [10] and [2].

In this paper, we consider an infinite-horizon casino model. In view of the finding in [16], we also allow the gambler to use randomization. Apart from the analytical tractability, an infinite-horizon model can capture the situations where the gambler does not have a definite number of plays in mind before entering the casino. The approach of exhaustive search taken by [5] cannot work in this setting. In the present paper, we are able to find the strategies of a precommitted gambler and a naive gambler analytically, which allows us to build a general theoretical comparison of these strategies. To the best of our knowledge, our paper is the first to analytically study the strategies of a precommitted gambler and compare them with those of a naive gambler in a general discrete-time casino gambling setting and using CPT preferences.

We first investigate when the optimal strategy of the precommitted gambler does not exist and, in this case, find asymptotically optimal strategies. We further divide these asymptotically optimal strategies into three types: loss-exit, gain-exit, and nonexit strategies. In the original terms of [5] (for a five-period model), a loss-exit strategy is to exit at a fixed loss level and not to exit at any gain level, a gain-exit strategy is the opposite, and a nonexit strategy is not to stop at any gain and loss levels. In the present paper, as the time horizon is infinite and the optimal CPT value may not be attained, a loss-exit strategy is interpreted as exiting at a certain fixed loss level or at an asymptotically large gain level (i.e., the limiting strategy only stops at a loss, hence the name). Similar interpretations are applied to gain-exit and nonexit strategies. We find conditions under which the precommitted gambler takes loss-exit, gain-exit, and nonexit strategies, respectively, and our theoretical results are consistent with the numerical results in [5].

We then assume a piecewise power utility function and concave, power probability weighting functions. The concave, power weighting functions, although not typically used in CPT, are still able to model the overweighing of very small probabilities of large gains and losses. With this specification of the utility and weighting functions, we are able to derive the optimal strategy of the precommitted gambler completely and analytically. The optimal strategy, when it exists, is to stop gambling when $[32]$.

${ }^{1}$ The classification of these three types of agents when facing time-inconsistency dates back to 
reaching a random level of gains or a random level of losses, and the latter level takes no more than two values and thus the optimal strategy yields a limited loss.

Finally, we study the actual behavior of a naive gambler and compare it with that of the precommitted gambler. We find the conditions under which the naive gambler stays at the casino forever. In particular, we prove that under some conditions, while the precommitted gambler will stop with probability one before the loss hits a certain level, the naive gambler continues to play with a positive probability at any loss level.

The explanation for the above gambling behavior, which was first provided by [5], is as follows: for the most relevant CPT parameter values, the preference value of taking a risk that yields a huge loss, e.g., $\$ 1000$, with a small probability, e.g., $0.1 \%$, is very negative because the weighting of this loss is strong due to the gambler's large sensitivity with respect to probability changes near the left end of the probability scale. Thus, the gambler does not want to end up with a huge loss and thus plans to stop with probability one before the loss hits a certain level. When the gambler keeps losing money and reaches the loss level that is supposed to trigger the stopping time under her initial plan, the probability of reaching this loss level is no longer small and thus this loss level is no longer heavily weighted. Consequently, she may find that it is optimal not to stop. Moreover, the above explanation predicts that the risk-taking of the gambler is greater after a paper loss than before the paper loss. This prediction is consistent with the experimental studies by [6], [3], and [23].

Mathematically, the casino gambling problem for a precommitted gambler in our paper is inherently a discrete-time optimal stopping problem in which the underlying state process is a symmetric random walk and the objective function is represented by CPT. Our mathematical contribution is to solve this optimal stopping problem analytically. We note that optimal stopping problems in a continuous-time setting with CPT preferences have been studied by [36] and [20], but such problems are different from our setting both in terms of their key features as well as the methods used to solve them. First, from the methodological point of view, $\mathrm{Xu}$ and Zhou [36] solve an optimization problem with the decision variable being the quantile function of the stopped state value. This technique of "quantile formulation" is later also used by [20]. In the discrete-time setting of our paper, such a quantile function is integer-valued because the state process in our model, the symmetric random walk, takes integer values. Consequently, the set of feasible quantile functions is nonconvex, which makes this approach inefficient, if not impossible. Instead, the approach we take is to solve an optimization problem with the decision variable to be the distribution function of the stopped state value. Second, from the point of view of stylized model features, the continuous time setting allows the gambler to construct arbitrarily small random payoffs. This was key to the finding in [11] that a naive agent never stops gambling, a finding which has been complemented and countered by [19] with the introduction of randomization; see section 5 for further discussion. In our model the stake size is fixed at $\$ 1$ and the gambler cannot construct arbitrarily small payoffs. In addition, the aforementioned two papers assume either that the gambler's utility function for gains and losses has finite left- and right-derivatives at the break-even point or that the utility function is piecewise power with the same diminishing sensitivity for gains and losses, whereas in the present paper we consider a more general utility function. Finally, we mention another recent work of Huang, Nguyen-Huu, and Zhou [22], who consider the optimal selling of a stock for an agent with rank-dependent utility (see e.g., [31]) and study the stopping behaviors of a naive agent. Again, their methods are linked to the continuous time setting in which the stock price follows a geometric Brownian motion and are not compatible with our discrete time setup. 
In the five-period model considered by [5], the strategy of a sophisticated gambler without precommitment is defined through a backward induction: starting from the end of the time horizon when the only choice of the gambler is to leave the casino, at the beginning of each period, the gambler decides her strategy knowing what her action will be in the following periods. In our model, such a definition is impossible because the end of the infinite time horizon does not exist. We can consider the game-theoretical approach to time-inconsistent problems, in which the agent's selves at different time are regarded as different players in a sequential game and an equilibrium strategy is sought; see, for instance, [32] and [29]. This approach has been used by [12] and [22] to study the behavior of a sophisticated agent without commitment in a continuous-time optimal stopping problem in which the agent's preferences involve probability weighting. In particular, Ebert and Strack [12] provide a sufficient condition under which gambling is never an equilibrium strategy for an agent with CPT preferences. The analysis of equilibrium strategies in our discrete-time model is much more involved than that in [12] and [22]; in particular, in the latter never starting to play is always an equilibrium strategy, but it is not the case in the discrete-time model. Therefore, we opt not to discuss sophisticated gamblers without commitment in our model and leave the study of such gamblers as future research.

The remainder of this paper is organized as follows. In section 2, we propose the casino gambling model. In section 3, we study the existence of the optimal strategies and discuss loss-exit, gain-exit, and nonexit strategies. In section 4, we solve the gambling problem completely when the utility function is piecewise power and the probability weighting functions are power. We then discuss the strategies taken by a naive gambler in section 5 . Finally, section 6 concludes. Technical and lengthy arguments for solving the underlying infinite-dimensional program, namely, the proofs of all results in section 4 , are relegated to online supplementary materials (available at SSRN.2682637). All the other proofs are placed in the appendix.

2. The model. We consider the casino gambling problem initially formulated by [5] but in an infinite-time setting. At time 0, a gambler is offered a fair bet, e.g., roulette wheel, in a casino: win or lose one dollar with equal probability. If the gambler takes the bet, the bet outcome is played out at time 1 . Then the gambler is offered the same bet and she decides whether to play. The same bet is offered and played out repeatedly until the first time the gambler declines the bet. At that time, the gambler leaves the casino with all her prior gains and losses. The gambler needs to decide the time $\tau$ to exit the casino.

Denote by $S_{t}, t=0,1, \ldots$ the cumulative gain or loss of the gambler in the casino at $t$. Then, $\left\{S_{t}: t \geq 0\right\}$ is a symmetric random walk. The gambler chooses the exit time $\tau$ to maximize her preference for $S_{\tau}$, while the preferences are modeled by CPT, assuming the reference point to be her initial wealth at the time she enters the casino. Then, the gambler's preference value of $S_{\tau}$ is

$$
\begin{aligned}
V\left(S_{\tau}\right)= & \sum_{n=1}^{\infty} u_{+}(n)\left(w_{+}\left(\mathbb{P}\left(S_{\tau} \geq n\right)\right)-w_{+}\left(\mathbb{P}\left(S_{\tau}>n\right)\right)\right) \\
& -\sum_{n=1}^{\infty} u_{-}(n)\left(w_{-}\left(\mathbb{P}\left(S_{\tau} \leq-n\right)\right)-w_{-}\left(\mathbb{P}\left(S_{\tau}<-n\right)\right)\right),
\end{aligned}
$$

where $u_{+}(\cdot)$ and $u_{-}(\cdot)$ are the utility function of gains and disutility function of losses, respectively, and $w_{+}(\cdot)$ and $w_{-}(\cdot)$ are the probability weighting functions regarding gains and losses, respectively. Following CPT, we assume that $u_{ \pm}(\cdot)$ are continuous, increasing, and concave and satisfy $u_{ \pm}(0)=0$. In addition, $w_{ \pm}(\cdot)$ are increasing 
mappings from $[0,1]$ onto $[0,1]$. We adopt the convention that $\infty-\infty=-\infty$ so that $V\left(S_{\tau}\right):=-\infty$ if the second sum of infinite series in $(2.1)$ is $+\infty$.

He et al. [16] shows that external randomization, such as tossing coins, can strictly improve the gambler's preference value. Moreover, individuals indeed use randomization in various contexts; see, e.g., [10] and [2]. Thus, different from [5], we allow the gambler to take randomized strategies. Importantly, the gambler can only use uniformly integrable stopping times $\tau$, i.e., $\left\{S_{\tau \wedge t}\right\}, t=0,1, \ldots$ needs to be uniformly integrable, so as to, among other things, exclude doubling strategies. Further, with no loss of generality (see section 4.1 for details), we can restrict the gambler to using either randomized path-independent stopping times or randomized Azéma-Yor stopping times. Both of these types of randomized stopping times involve tossing coins and are easy to implement (see [17]).

Denote by $\mathcal{T}$ the set of feasible stopping times described above. Then, the gambler faces the following optimization problem at time 0 :

$$
\max _{\tau \in \mathcal{T}} V\left(S_{\tau}\right)
$$

The introduction of randomization, in combination with the infinite-time horizon setting, enables us to apply the techniques of Skorohod embedding from [17], which is a crucial step to solve (2.2); see section 4 below. ${ }^{2}$

As shown in [5], when the gambler uses the same CPT preferences to revisit the gambling problem in the future, e.g., at time $t>0$, the optimal strategy of problem (2.2), which is solved at time 0 , is no longer optimal. This arises from the probability weighting function. If the gambler commits her future selves to the strategy solved at time 0 , the optimal strategy of problem (2.2) is the one that is implemented by the gambler. In this case, the gambler is (sophisticated and) precommitted. If at each time the gambler is unaware of the time-inconsistency, she may keep changing her strategy in the future. In this case, the gambler is naive, and the actual strategy implemented by the gambler is different from the optimal strategy of problem (2.2). In the following, we first study the precommitting strategy, i.e., the optimal strategy of the precommitted gambler, in sections 3 and 4 . Then, we study the actual behavior of a naive gambler in section 5 .

The following usual notation will be used throughout the paper: for any real number $x,\lfloor x\rfloor$ stands for the floor of $x$, i.e., the largest integer dominated by $x$, and $\lceil x\rceil$ stands for the ceil of $x$, i.e., the smallest integer dominating $x$. For real numbers $x$ and $y, x \wedge y:=\min (x, y)$.

3. Loss-exit, gain-exit, and nonexit strategies. In this section, we study various behaviors of precommitted gamblers via a class of simple two-level strategies. For two integers $b<0<a$ we denote by $\tau_{a, b}$ the first exit time from $[b, a]$, i.e., $\tau_{a, b}:=\inf \left\{t \geq 0: S_{t}=a\right.$ or $\left.S_{t}=b\right\}$.

THEOREM 3.1. Let $V^{*}$ be the value of the optimization problem in (2.2).

(i) Suppose there exists $s>0$ such that

$$
\liminf _{x \rightarrow+\infty} u_{+}(s x) w_{+}(1 / x)=+\infty .
$$

Then, $V^{*}=\infty$ and there exists $b<0$ such that $\lim _{a \rightarrow+\infty} V\left(S_{\tau_{a, b}}\right)=+\infty$.

\footnotetext{
${ }^{2}$ The Skorokhod embedding, in our context, states that for any zero-mean, integrable distribution on $\mathbb{Z}$, there exists a uniformly integrable, randomized stopping time $\tau$ such that $S_{\tau}$ follows that distribution. If randomization is not allowed, such a result does not hold unless we do not assume uniform integrability of $\tau$; see, e.g., [9]. The above Skorokhod embedding result does not hold either in a finite-horizon setting.
} 
(ii) Suppose there exists $\epsilon>0$ such that

$$
\limsup _{x \rightarrow+\infty} u_{-}\left(x^{1+\epsilon}\right) w_{-}(1 / x)=0
$$

Then, $V^{*}=\sup _{x>0} u_{+}(x)$. Moreover, the optimal solution to problem (2.2) does not exist and $V\left(S_{\tau_{a, b}}\right)$ with $a=\left\lfloor k^{\epsilon /(1+\epsilon)}\right\rfloor$ and $b=-k$ converges to $V^{*}$ as $k$ goes to infinity.

(iii) Suppose $\lim _{x \rightarrow+\infty} u_{+}(x)=+\infty$, and there exists $p \in(0,1)$ such that

$$
\limsup _{x \rightarrow+\infty} \frac{u_{-}\left(\frac{p}{1-p} x\right)}{u_{+}(x)}<\frac{w_{+}(p)}{w_{-}(1-p)} .
$$

Then, $V^{*}=\infty$ and there exists $c>0$ such that $\lim _{k \rightarrow+\infty} V\left(S_{\tau_{a, b}}\right)=+\infty$, where $a=\lceil c k\rceil$ and $b=-k$.

Note that the above result only uses two-level strategies, which are among the simplest possible strategies. In particular, Theorem 3.1 still holds even if the gambler is restricted to using, e.g., only nonrandomized or path-independent strategies.

Theorem 3.1 provides three cases in which the optimal solution to problem (2.2) does not exist. ${ }^{3}$ Note that the three cases are not mutually exclusive; see Table 1 and the associated discussion below. In these cases, we are still able to study the gambler's behavior by investigating the strategies taken by the gambler to reach the optimal value asymptotically. ${ }^{4}$ In the first case, (i), the optimal value of the gambling problem (2.2) is infinite. The strategy $\tau_{a, b}$ the gambler takes, as $a \rightarrow+\infty$, is of the "stop-lossand-let-profit-run" type: to exit once reaching a fixed loss level $-b$ and not to stop in gain. It is essentially a loss-exit strategy, in Barberis's term; see [5]. Such a strategy caps the loss and allows infinite gain, producing a highly skewed distribution which is favored due to probability weighting. To interpret the assumption (3.1), consider the following lottery: winning $s x$ dollars with probability $1 / x$ and winning nothing otherwise, where $x$ is a sufficiently large number. The expected payoff of the lottery is $s$ and the CPT value of the lottery is $u_{+}(s x) w_{+}(1 / x)$. Therefore, condition (3.1) implies that the gambler has strong preferences for this type of highly skewed lotteries so that with limited wealth $s$, she can achieve infinite CPT value by purchasing these lotteries.

In case (ii), the optimal value is finite or infinite depending on whether or not $u_{+}$ is uniformly bounded; however, either way this value is not attained by any stopping strategy. The gambler likes the following strategy: leave the casino when losing more than $k$ dollars or when winning more than $\left\lfloor k^{\epsilon /(1+\epsilon)}\right\rfloor$ dollars for sufficiently large $k$. Note that $\left\lfloor k^{\epsilon /(1+\epsilon)}\right\rfloor$ is much smaller than $k$ when $\epsilon$ is small. For instance, when $\epsilon=0.25$ and $k=100000$, the strategy taken by the gambler is to stop gambling when winning $\$ 10$ or when losing $\$ 100000$. To understand the preferences underlying this

\footnotetext{
${ }^{3}$ The optimal value for the precommitted gambler may or may not be infinite. For example, in case (ii) of Theorem 3.1, the optimal value is finite if $\sup _{x>0} u_{+}(x)<+\infty$. When the optimal value is not attainable, the problem is said to be ill-posed.

${ }^{4} \mathrm{He}$ and Zhou [18, p. 316] consider a single-period portfolio selection problem under CPT and argue that "an ill-posed model is one whose optimal strategy is simply to take the greatest possible risky exposure". Jin and Zhou [24] and [25] consider a continuous-time portfolio selection problem under CPT and find that for certain parameter values, the problem is ill-posed. Although the authors were critical of the ill-posedness in these papers, following their analysis one can also find the strategies that reach the unattainable optimal value asymptotically, and this can tell us the agent's behavior even when the problem is ill-posed.
} 
sort of behavior, consider the following random loss: losing $x^{1+\epsilon}$ dollars with $1 / x$ probability and losing nothing otherwise, where $x$ is sufficiently large. The expected loss is as large as $x^{\epsilon}$. However, condition (3.2) implies that the CPT value of this random loss, $-u_{-}\left(x^{1+\epsilon}\right) w_{-}(1 / x)$, is nearly zero. Hence, the above strategy is a result of the gambler not willing to pay anything to hedge against the loss, even though the expected loss is large. It corresponds essentially to a gain-exit strategy in [5].

Azevedo and Gottlieb [4] propose two conditions in Proposition 1 therein and show that under these two conditions, a risk-neutral firm can extract unbounded profits from consumers with prospect theory preferences. Conditions (3.1) and (3.2) in the present paper resemble those in [4].

In case (iii) of Theorem 3.1, condition (3.3) is related to the large loss aversion degree (LLAD), defined as $\lim _{x \rightarrow+\infty} u_{-}(x) / u_{+}(x)$, in [18]. Indeed, by setting $p=1 / 2$, condition (3.3) is implied by the one that LLAD is strictly less than $w_{+}(1 / 2) / w_{-}(1 / 2)$, i.e., LLAD is sufficiently low. In this case, condition (3.3) implies that probability weighting on large gains dominates a combination of weighting on large losses and loss aversion. The corresponding strategy taken by the gambler is to exit when either the gain reaches a sufficiently high level $(\lfloor c k\rfloor)$ or the loss reaches another sufficiently high level $(k)$, and these two levels are of the same magnitude. Therefore, we can consider it to be an essentially nonexit strategy.

We can summarize the above discussions conveniently using a single quantity. Namely, for any stopping time $\tau \in \mathcal{T}$, define the conditional gain-loss ratio as

$$
R(\tau):=\frac{\mathbb{E}\left[S_{\tau} \mid S_{\tau} \geq 1\right]}{\mathbb{E}\left[-S_{\tau} \mid S_{\tau} \leq-1\right]} .
$$

For stopping time $\tau_{a, b}$, we have $R\left(\tau_{a, b}\right)=a /(-b)$. Thus, Theorem 3.1(i) corresponds to an arbitrarily large conditional gain-loss ratio, while Theorem 3.1(ii) corresponds to an arbitrarily small conditional gain-loss ratio. Finally, for $\tau_{a, b}$ with $a=\lceil c k\rceil$, $b=-k$, and $k \rightarrow \infty$ as in Theorem 3.1(iii), we can see that $R\left(\tau_{a, b}\right)$ is approximately a positive constant $c$ and the conditional expected gain $\mathbb{E}\left[S_{\tau} \mid S_{\tau} \geq 1\right]$ is arbitrarily large.

Next, we consider the following specification of the utility function and probability weighting functions introduced by [33]:

$$
u_{+}(x)=x^{\alpha_{+}}, \quad u_{-}(x)=\lambda x^{\alpha_{-}}, \quad w_{ \pm}(p)=\frac{p^{\delta_{ \pm}}}{\left(p^{\delta_{ \pm}}+(1-p)^{\delta_{ \pm}}\right)^{1 / \delta_{ \pm}}},
$$

where $\alpha_{+}, \alpha_{-} \in(0,1]$ and $\delta_{+}, \delta_{-} \in(0,1]$ such that $w_{ \pm}(\cdot)$ are increasing. With this specification, the results of Theorem 3.1 are summarized in Table 1 . These results also hold in the case of power probability weighting functions $w_{ \pm}(p)=p^{\delta_{ \pm}}$and in the case of the probability weighting functions proposed by [15]:

$$
w_{ \pm}(p)=a_{ \pm} p^{\delta_{ \pm}} /\left(a_{ \pm} p^{\delta_{ \pm}}+(1-p)^{\delta_{ \pm}}\right)
$$

with $a_{ \pm} \in(0,1]$.

As noted previously, the conditions (3.1)-(3.3), which correspond to the four rows in Table 1, are not exclusive to each other in general. For example, it is possible that both $\alpha_{+}>\delta_{+}$and $\alpha_{-}<\delta_{-}$are true, in which case both the gain-exit strategy and the loss-exit strategy can lead to the optimal preference value. However, if we take the parameter values used in [5], i.e., (3.4) with $\alpha_{+}=\alpha_{-}=\alpha$ and $\delta_{+}=\delta_{-}=\delta$, then the first two rows in Table 1 are mutually exclusive. We show analytically that the gambler takes loss-exit strategies when $\alpha>\delta$ and takes gain-exit strategies when 
TABLE 1

Existence of optimal solutions. The utility function is given as in (3.4), and the weighting functions are given as in (3.4), in (3.5), or in power functions $w_{ \pm}(p)=p^{\delta_{ \pm}}$.

\begin{tabular}{l|c|l}
\hline Parameter values & Exist. opt. solution & Strategy \\
\hline$\alpha_{+}>\delta_{+}$ & No & loss-exit \\
$\alpha_{-}<\delta_{-}$ & No & gain-exit \\
$\alpha_{+}>\alpha_{-}$ & No & nonexit \\
$\alpha_{+}=\alpha_{-}, \lambda<\sup _{0<p<1} \frac{w_{+}(p) / p^{\alpha}}{w_{-}(1-p) /(1-p)^{\alpha}}$ & No & nonexit \\
\hline
\end{tabular}

$\alpha<\delta$. These theoretical results coincide with the numerical results presented by $[5$, Figure 3] in the simple five period setting therein, but they are valid more generally.

For weighting function

$$
w_{ \pm}(p)=e^{-a_{ \pm}(-\ln p)^{\delta} \pm}
$$

with $a_{ \pm}>0$ and $\delta_{ \pm} \in(0,1]$, which was proposed by [30], condition (3.1) is satisfied for any $\delta_{+}<1$ or for $\delta_{+}=1$ and $\alpha_{+}>a_{+}$. In this case, the gambler takes an asymptotically loss-exit strategy. Condition (3.2), however, is not satisfied for any $\delta_{-}<1$.

Finally, we investigate whether the experimental estimates of the utility function and probability weighting functions imply any of the loss-exit, gain-exit, and nonexit strategies in our model. As long as $\delta_{ \pm} \neq 1$, the above analysis shows that with probability weighting function (3.6), the gambler takes the loss-exit but not the gainexit strategy. In the following, we focus on the experimental estimates of the CPT preferences in the literature using probability weighting function (3.4) or (3.5). We plot in Figure 1 the estimates of $\alpha_{ \pm}$for the utility function as in (3.4) and the estimates of $\delta_{ \pm}$for the probability weighting function as in (3.4) or for the one in (3.5): In the left pane $\alpha_{+}$versus $\delta_{+}$is plotted, in the left-middle pane $\delta_{-}$versus $\alpha_{-}$is plotted, in the right-middle pane $\alpha_{+}$versus $\alpha_{-}$is plotted, and in the right pane $\delta_{+}$versus $\delta_{-}$ is plotted. ${ }^{5}$ We observe that for most of the estimates in the literature, $\alpha_{+}<\alpha_{-}$, $\alpha_{-}>\delta_{-}$, and $\alpha_{+}>\delta_{+}$. Hence, our general results imply that, for the most relevant parameter values, the precommitted gambler takes the loss-exit strategy but not the gain-exit or the nonexit strategy.

It is, however, still of interest to study the precommitted gambler's strategy when Theorem 3.1 does not apply because in two of the experimental studies listed in Figure 1 , we do have $\alpha_{+}<\delta_{+}$. Thus, in the next section, we plan to solve problem (2.2), assuming that none of the conditions in Theorem 3.1 apply which, as will be clear in the next section, "nearly" implies that an optimal solution exists.

4. Precommitted gamblers with power utility and weighting functions. In this section, we solve problem (2.2) for a precommitted gambler when the utility function is piecewise power and the probability weighting functions are power

\footnotetext{
${ }^{5}$ We consider only experiments that involve monetary payoffs under risk, as they are relevant to our casino gambling problem. In addition, we consider only experiments that treat $\alpha_{+}$and $\alpha_{-}$ separately, treat $\delta_{+}$and $\delta_{-}$separately, and estimate the utility function and probability weighting function simultaneously. This leads to seven works we are aware of. Some works estimate $\alpha_{+}$and $\delta_{+}$ only, and some others estimate $\alpha_{-}$and $\delta_{-}$only. When the experiment in a work involves multiple groups of subjects or multiple sessions, we use the grand estimate. Some of the works estimate the probability weighting functions both in (3.4) and (3.5), but the estimates are very similar so we simply take the average. Finally, when both the mean and median of the estimate of a parameter are available, we select the median.
} 

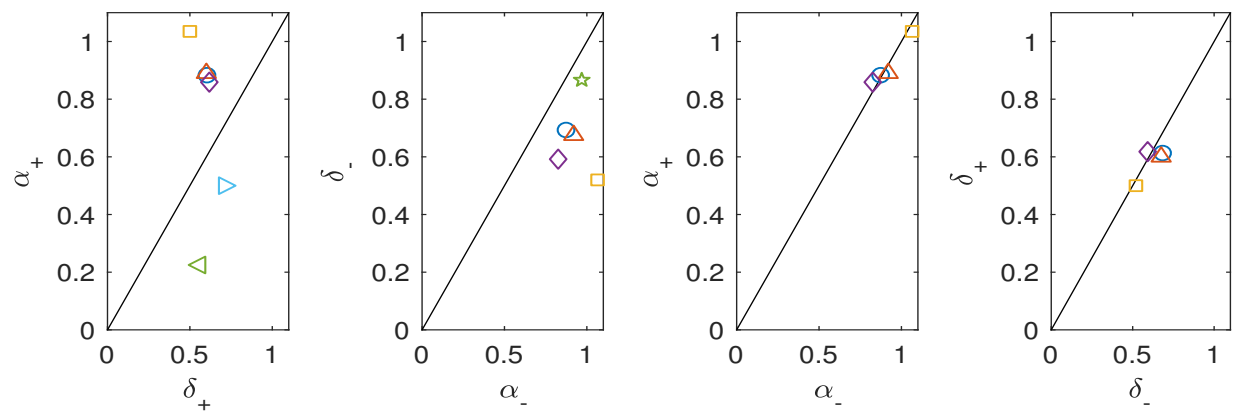

FIG. 1. Experimental estimates of $\alpha_{ \pm}$for the utility function as in (3.4) and $\delta_{ \pm}$for the probability weighting function as in (3.4) or as in (3.5). The left pane plots $\alpha_{+}$versu $\delta_{+}$, the left-middle pane plots $\delta_{-}$versus $\alpha_{-}$, the right-middle pane plots $\alpha_{+}$versus $\alpha_{-}$, and the right pane plots $\delta_{+}$versus $\delta_{-}$. The estimate $\left(\alpha_{+}, \alpha_{-}, \delta_{+}, \delta_{-}\right)=(0.88,0.88,0.61,0.69)$ by [33] is represented by the circle shape, the estimate $\left(\alpha_{+}, \alpha_{-}, \delta_{+}, \delta_{-}\right)=(0.89,0.92,0.60,0.675)$ by $[1]$ is represented by the facing-up triangle shape, the estimate $\left(\alpha_{+}, \alpha_{-}, \delta_{+}, \delta_{-}\right)=(1.035,1.065,0.5,0.52)$ by [14] is represented by the square shape, the estimate $\left(\alpha_{+}, \alpha_{-}, \delta_{+}, \delta_{-}\right)=(0.859,0.826,0.618,0.592)$ by [7] is represented by the diamond shape, the estimate $\left(\alpha_{+}, \delta_{+}\right)=(0.225,0.56)$ by [8] is represented by the facing-left triangle shape, the estimate $\left(\alpha_{+}, \delta_{+}\right)=(0.5,0.71)$ by [35] is represented by the facing-right triangle shape, and the estimate $\left(\alpha_{-}, \delta_{-}\right)=(0.969,0.8665)$ by [13] is represented by the pentagram shape.

functions, i.e., when

$$
u_{+}(x)=x^{\alpha_{+}}, \quad u_{-}(x)=\lambda x^{\alpha_{-}}, \quad w_{+}(p)=p^{\delta_{+}}, \quad w_{-}(p)=p^{\delta_{-}}
$$

with $\alpha_{ \pm} \in(0,1], \delta_{ \pm} \in(0,1]$, and $\lambda>0$. The parameters $\alpha_{+}$and $\alpha_{-}$measure the diminishing sensitivity of the utility of gains and losses, respectively; the smaller $\alpha_{+}$ and $\alpha_{-}$are, the more risk averse regarding gains and the more risk seeking regarding losses the gambler is, respectively.

The parameter $\lambda$ is related to loss aversion, a phenomenon in which individuals are more sensitive to losses than to gains; see, for instance, [26]. Various definitions of loss aversion degree, a measure of the extent to which individuals are averse to losses, have been proposed in the literature. These include the function $x \rightarrow u_{-}(x) / u_{+}(x)$ in [27], the constant $u_{-}(1) / u_{+}(1)$ in [33], the function $x \rightarrow u_{-}^{\prime}(x) / u_{+}^{\prime}(x)$ in [34], the value of $\lim _{x \downarrow 0} u_{-}(x) / u_{+}(x)$ in [28], and that of $\lim _{x \uparrow+\infty} u_{-}(x) / u_{+}(x)$ in [18]. Except for the definition in [33], the parameter $\lambda$ in (4.1) does not coincide with the other definitions unless $\alpha_{+}=\alpha_{-}$. In addition, the definition of loss aversion degree by [33] depends on the choice of the unit of monetary payoffs, e.g., using dollars or euros as the units lead to different loss aversion degree in this definition. Therefore, $\lambda$, although related to loss aversion, cannot be directly interpreted as the loss aversion degree. However, with $\alpha_{+}$and $\alpha_{-}$being fixed, the larger $\lambda$ is, the more loss averse and hence the more overall risk averse the gambler is.

Note that a commonly used probability weighting function supported by empirical observations is inverse-S-shaped, i.e., is concave in its low end and convex in its high end. The power probability weighting functions in (4.1), however, are concave. We consider the power instead of inverse-S-shaped probability weighting functions since this makes tractable solutions possible while maintaining the key stylized feature of modeling individuals' tendency to overweight the tails of payoff distributions. Indeed, in our gambling problem, the cumulative gain or loss represents a mixed gamble for which both gains and losses are possible. The tails therefore refer to significant gains 
and losses of small probability. The tendency to overweight these is captured by the concave parts in the low ends of the two probability weighting functions (see the CPT preference value (2.1)) and is achieved with the two power probability weighting functions. We can see that the smaller $\delta_{+}$and $\delta_{-}$are, the more the gambler overweighs large gains and losses of small probability, respectively.

In the remainder of this section, we always assume (4.1) for the utility and probability weighting functions. Because of the results in Table 1 , we only need to consider the following parameter ranges, $\alpha_{+} \leq \delta_{+}, \alpha_{-} \geq \delta_{-}$, and $\alpha_{+} \leq \alpha_{-}$, throughout this section. As discussed at the end of section 3 , although the parameter range $\alpha_{+}<\delta_{+}$ is not the most commonly observed one in the experimental estimates of CPT parameters, it is still of interest to study the optimal gambling behavior in this case.

4.1. Infinite-dimensional programming. A key step is to turn the original problem (2.2) into an infinite-dimensional program that is tractable. For any uniformly integrable stopping time $\tau, \mathbb{E}\left[S_{\tau}\right]=\lim _{t \rightarrow \infty} \mathbb{E}\left[S_{\tau \wedge t}\right]=0$. Thus, the distribution of $S_{\tau}$ is a centered probability measure on $\mathbb{Z}$, i.e., the distribution is integrable and has zero mean. On the other hand, as shown by [17], any centered probability measure $\mu$ on $\mathbb{Z}$ can be embedded into $\left\{S_{t}\right\}$ both by a uniformly integrable randomized path-independent stopping time and by a uniformly integrable randomized AzémaYor stopping time, i.e., $\left\{S_{t}\right\}$ at the stopping time follows $\mu$. Thus, to solve the optimal stopping problem (2.2), we can first find the optimal centered probability measure for $\left\{S_{t}\right\}$ at the stopping time and then use the Skorohod embedding results in [17] to compute the stopping time corresponding to the optimal probability measure.

Use $\boldsymbol{x}:=\left(x_{1}, x_{2}, \ldots\right)$ and $\boldsymbol{y}:=\left(y_{1}, y_{2}, \ldots\right)$ to stand for the decumulative distribution of the gain and the cumulative distribution of the loss, respectively, of $S_{\tau}$ for some stopping time $\tau \in \mathcal{T}$, i.e., $x_{n}=\mathbb{P}\left(S_{\tau} \geq n\right)$ and $y_{n}=\mathbb{P}\left(S_{\tau} \leq-n\right), n \geq 1$. Then, the objective function in (2.2) can be written as

$$
U(\boldsymbol{x}, \boldsymbol{y}):=\sum_{n=1}^{\infty}\left(u_{+}(n)-u_{+}(n-1)\right) w_{+}\left(x_{n}\right)-\sum_{n=1}^{\infty}\left(u_{-}(n)-u_{-}(n-1)\right) w_{-}\left(y_{n}\right)
$$

with $U(\boldsymbol{x}, \boldsymbol{y}):=-\infty$ if the second sum of infinite series is infinity. Here, the form of $U(\boldsymbol{x}, \boldsymbol{y})$ is obtained by applying Fubini's theorem to (2.1). Consequently, problem $(2.2)$ is equivalent to the following infinite-dimensional optimization problem:

$$
\begin{array}{cl}
\max _{\boldsymbol{x}, \boldsymbol{y}} & U(\boldsymbol{x}, \boldsymbol{y}) \\
\text { subject to } & 1 \geq x_{1} \geq x_{2} \geq \cdots \geq x_{n} \geq \cdots \geq 0 \\
& 1 \geq y_{1} \geq y_{2} \geq \cdots \geq y_{n} \geq \cdots \geq 0 \\
& x_{1}+y_{1} \leq 1, \sum_{n=1}^{\infty} x_{n}=\sum_{n=1}^{\infty} y_{n}
\end{array}
$$

where the first three constraints are present because $\boldsymbol{x}$ and $\boldsymbol{y}$ are the decumulative and cumulative distributions of the gain and loss, respectively, of $S_{\tau}$ and the last constraint is the case because the distribution of $S_{\tau}$ is centered, i.e., $\mathbb{E}\left[S_{\tau}\right]=0$.

It is worth noting that because $S_{0}=0$, exit time $\tau=0$, which means not to gamble, corresponds to $\boldsymbol{x}=\boldsymbol{y}=0$, and the objective value $U(\boldsymbol{x}, \boldsymbol{y})=0$. Therefore, the gambler chooses to gamble if and only if there exist $\boldsymbol{x}$ and $\boldsymbol{y}$ such that $U(\boldsymbol{x}, \boldsymbol{y})>0$.

4.2. A decomposition. Problem (4.2) is difficult to solve for two main reasons. First, the objective function is neither concave nor convex in the decision variable because both $w_{+}(\cdot)$ and $w_{-}(\cdot)$ are concave. Second, there are infinitely many constraints, so the standard Lagrange dual method is not directly applicable even if the objective 
function were concave. To overcome the difficulty, we first decompose problem (4.2) into two subproblems.

For any $\boldsymbol{x}$ and $\boldsymbol{y}$ of problem (4.2) corresponding to some stopping time $\tau$, introduce $s_{+}:=\left(\sum_{n=2}^{\infty} x_{n}\right) / x_{1}$ and $s_{-}:=\left(\sum_{n=2}^{\infty} y_{n}\right) / y_{1}$. Then,

$$
s_{+}=\left(\sum_{n=2}^{\infty} \mathbb{P}\left(S_{\tau} \geq n\right)\right) / \mathbb{P}\left(S_{\tau} \geq 1\right)=\mathbb{E}\left[S_{\tau}-1 \mid S_{\tau} \geq 1\right] .
$$

Similarly, $s_{-}=\mathbb{E}\left[-S_{\tau}-1 \mid S_{\tau} \leq-1\right]$. On the other hand, define $\boldsymbol{z}_{+}=\left(z_{+, 1}, z_{+, 2}, \ldots\right)$ with $z_{+, n}:=x_{n+1} / x_{1}, n \geq 1$, and $\boldsymbol{z}_{-}=\left(z_{-, 1}, z_{-, 2}, \ldots\right)$ with $z_{-, n}:=y_{n+1} / y_{1}, n \geq 1$. Note that $\sum_{n=1}^{\infty} z_{ \pm, n}=s_{ \pm}$.

In order to find the optimal $\boldsymbol{x}$ and $\boldsymbol{y}$, we only need first to find the optimal $\boldsymbol{z}_{+}$ and $\boldsymbol{z}_{-}$with constraint $\sum_{n=1}^{\infty} z_{ \pm, n}=s_{ \pm}$, and then to find the optimal $x_{1}, y_{1}$, and $s_{ \pm}$. Furthermore, $s_{ \pm}$imply the conditional expected gain and loss of $S_{\tau}$ and the conditional-gain loss ratio, i.e.,

$$
\mathbb{E}\left[S_{\tau} \mid S_{\tau} \geq 1\right]=s_{+}+1, \quad \mathbb{E}\left[-S_{\tau} \mid S_{\tau} \leq-1\right]=s_{-}+1, \quad R(\tau)=\frac{s_{+}+1}{s_{-}+1} .
$$

Therefore, the (asymptotically) optimal value $s_{ \pm}$will dictate whether the gambler takes a loss-exit, gain-exit, or nonexit strategy. Finally, after we find optimal $\boldsymbol{z}_{ \pm}, x_{1}$, $y_{1}$, and $s_{ \pm}$, we can compute optimal $\boldsymbol{x}$ and $\boldsymbol{y}$ as $\boldsymbol{x}=\left(x_{1}, x_{1} \boldsymbol{z}_{+}\right)$and $\boldsymbol{y}=\left(y_{1}, y_{1} \boldsymbol{z}_{-}\right)$, respectively.

The above procedure can be summarized as the following three problems. First, for each fixed $s \geq 0$, we consider the following gain-part problem,

$$
\begin{array}{cl}
\underset{z}{\operatorname{Max}} & \sum_{n=1}^{\infty}\left((n+1)^{\alpha_{+}}-n^{\alpha_{+}}\right)\left(z_{n}\right)^{\delta_{+}} \\
\text {subject to } & 1 \geq z_{1} \geq z_{2} \geq \cdots \geq z_{n} \geq \cdots \geq 0 \\
& \sum_{n=1}^{\infty} z_{n}=s
\end{array}
$$

and denote by $v_{+}(s)$ the optimal value of this problem. Second, we consider the following loss-part problem,

$$
\begin{array}{cl}
\operatorname{Min}_{\boldsymbol{z}} & \sum_{n=1}^{\infty}\left((n+1)^{\alpha_{-}}-n^{\alpha_{-}}\right)\left(z_{n}\right)^{\delta_{-}} \\
\text {subject to } & 1 \geq z_{1} \geq z_{2} \geq \cdots \geq z_{n} \geq \cdots \geq 0, \\
& \sum_{n=1}^{\infty} z_{n}=s,
\end{array}
$$

and denote its optimal value as $v_{-}(s)$. Finally, we consider the master problem

$$
\begin{array}{cl}
\underset{x_{1}, y_{1}, s_{+}, s_{-}}{\operatorname{Max}} & \left(v_{+}\left(s_{+}\right)+1\right) x_{1} \delta_{+}-\lambda\left(v_{-}\left(s_{-}\right)+1\right) y_{1}{ }_{-} \\
\text {subject to } & x_{1}+y_{1} \leq 1, \\
& \left(s_{+}+1\right) x_{1}=\left(s_{-}+1\right) y_{1}, \\
& x_{1} \geq 0, y_{1} \geq 0, s_{+} \geq 0, s_{-} \geq 0 .
\end{array}
$$

One can see that problems (4.2) and (4.5) have the same optimal value and the optimal solution to one of the problems exists if and only if that to the other exists. Moreover, suppose $\left(x_{1}^{*}, y_{1}^{*}, s_{+}^{*}, s_{-}^{*}\right)$ is optimal to problem (4.5) and $\boldsymbol{z}_{+}^{*}$ and $\boldsymbol{z}_{-}^{*}$ are optimal to problem (4.3) parameterized by $s_{+}^{*}$ and to problem (4.4) parameterized by $s_{-}^{*}$, respectively. Then, $\left(\boldsymbol{x}^{*}, \boldsymbol{y}^{*}\right)$ with $\boldsymbol{x}^{*}:=\left(x_{1}^{*}, x_{1}^{*} \boldsymbol{z}_{+}^{*}\right)$ and $\boldsymbol{y}^{*}:=\left(y_{1}^{*}, y_{1}^{*} \boldsymbol{z}_{-}^{*}\right)$ is 
optimal to problem (4.2). Therefore, to find the optimal solution to (4.2), we only need to solve each of the three problems (4.3)-(4.5).

Because solving problems (4.3)-(4.5) involves technical and lengthy arguments, we relegate all proofs in this section to the online supplementary materials (available at SSRN.2682637).

4.3. The gain-part problem. We first solve problem (4.3), which is an infinitedimensional optimization problem with an infinite number of constraints and thus difficult to solve. We present a complete solution in Proposition 4.1, and Proposition 4.2 provides several properties of the optimal value of problem (4.3), which will in turn be used in solving problem (4.5).

Proposition 4.1.

(i) When $\alpha_{+}=\delta_{+}=1$, any feasible solution to problem (4.3) is optimal and the optimal value $v_{+}(s)=s$.

(ii) When $\alpha_{+}<\delta_{+}=1$, the optimal solution is

$$
z_{n}^{*}=1, n=1, \ldots,\lfloor s\rfloor, z_{\lfloor s\rfloor+1}^{*}=s-\lfloor s\rfloor, z_{n}^{*}=0, n \geq\lfloor s\rfloor+2
$$

and the optimal value is

$$
v_{+}(s)=(1-(s-\lfloor s\rfloor))(\lfloor s\rfloor+1)^{\alpha_{+}}+(s-\lfloor s\rfloor)(\lfloor s\rfloor+2)^{\alpha_{+}}-1 .
$$

(iii) When $\alpha_{+}=\delta_{+}<1$, the optimal value of problem (4.3) is $+\infty$.

(iv) When $\alpha_{+}<\delta_{+}<1$, the optimal solution to problem (4.3) is

$$
z_{n}^{*}=\left[\mu(s)\left((n+1)^{\alpha_{+}}-n^{\alpha_{+}}\right)^{\frac{1}{1-\delta_{+}}}\right] \wedge 1, n=1,2, \ldots,
$$

where $\mu(s)>0$ is the number such that $\sum_{n=1}^{\infty} z_{n}^{*}=s$, and the optimal value is

$$
v_{+}(s)=\sum_{n=1}^{\infty}\left\{\left[\mu(s)^{\delta_{+}}\left((n+1)^{\alpha_{+}}-n^{\alpha_{+}}\right)^{\frac{1}{1-\delta_{+}}}\right] \wedge\left[(n+1)^{\alpha_{+}}-n^{\alpha_{+}}\right]\right\} .
$$

Proposition 4.2. Assume $\alpha_{+}<\delta_{+} \leq 1$.

(i) The optimal value function $v_{+}(\cdot)$ of problem (4.3) is continuous, strictly increasing, and concave in $[0,+\infty)$. If furthermore $\delta_{+}<1, v_{+}(\cdot)$ is continuously differentiable and strictly concave in $[0,+\infty)$.

(ii) There exist $C_{+}>c_{+}>0$ such that $c_{+}(s+1)^{\alpha_{+}} \leq v_{+}(s)+1 \leq C_{+}(s+1)^{\alpha_{+}}$ for all $s \geq 0$.

(iii) When $\alpha_{+}<\delta_{+}=1, g_{+}(s):=\left(v_{+}(s)+1\right) /(s+1)^{\delta_{+}}$is strictly decreasing in $[0,+\infty)$ and $g_{+}(0)=1$. When $\alpha_{+}<\delta_{+}<1, g_{+}(s)$ is strictly increasing in $\left[0, s_{1}\right]$ and strictly decreasing in $\left[s_{1},+\infty\right)$, where $s_{1}:=\sum_{n=1}^{\infty}\left((n+1)^{\alpha_{+}-}\right.$ $\left.n^{\alpha_{+}}\right)^{1 /\left(1-\delta_{+}\right)}$, and $g_{+}\left(s_{1}\right)=\left(1+s_{1}\right)^{1-\delta_{+}}$.

4.4. The loss-part problem. Next, we solve problem (4.4), again an infinitedimensional optimization problem with an infinite number of constraints. Proposition 4.3 in the following presents a complete solution to this problem. Further important properties of the optimal value function of problem (4.4), which are then used in solving problem (4.5), are established in Proposition 4.4.

Copyright $@$ by SIAM. Unauthorized reproduction of this article is prohibited. 


\section{Proposition 4.3.}

(i) Suppose $\alpha_{-}=\delta_{-}=1$. Then, any feasible solution to problem (4.4) is optimal and the optimal value $v_{-}(s)=s$.

(ii) Suppose $\delta_{-} \leq \alpha_{-}$and $\delta_{-}<1$. Define $B_{1}:=1$. For each integer $m \geq 2$, define $B_{m}=m$ when $\alpha_{-}=1$ and define $B_{m}$ to be the unique number in $(m-1, m)$ determined by

$\left((m+1)^{\alpha_{-}}-1\right)\left(\frac{B_{m}}{m}\right)^{\delta_{-}}=\left(m^{\alpha_{-}}-1\right)+\left((m+1)^{\alpha_{-}}-m^{\alpha_{-}}\right)\left(B_{m}-m+1\right)^{\delta_{-}}$

when $\alpha_{-}<1$. Then, for $s \in\left(m-1, B_{m}\right]$, the optimal solution to problem (4.4) is

$$
z_{n}^{*}=1, n=1, \ldots, m-1, z_{m}^{*}=s-m+1, z_{n}^{*}=0, n \geq m+1
$$

and the optimal value is

$$
v_{-}(s)=\left(m^{\alpha_{-}}-1\right)+\left((m+1)^{\alpha_{-}}-m^{\alpha_{-}}\right)(s-m+1)^{\delta_{-}} .
$$

For $s \in\left(B_{m}, m\right]$, the optimal solution to problem (4.4) is

$$
z_{n}^{*}=\frac{s}{m}, n=1, \ldots, m, z_{n}^{*}=0, n \geq m+1
$$

and the optimal value is

$$
v_{-}(s)=\left((m+1)^{\alpha_{-}}-1\right)\left(\frac{s}{m}\right)^{\delta_{-}} .
$$

Proposition 4.4. Assume $\delta_{-} \leq \alpha_{-}$and $\delta_{-}<1$. Recall $B_{m}, m \geq 1$ as defined in Proposition 4.3.

(i) The value function $v_{-}(s)$ of problem (4.4) is strictly increasing and continuous on $[0,+\infty)$, continuously differentiable in $\left(m-1, B_{m}\right) \cup\left(B_{m}, m\right)$, and strictly concave on $[m-1, m]$ for all positive integers $m$. Furthermore, when $B_{m}=m$, $\lim _{s \uparrow m} v_{-}^{\prime}(s)<\lim _{s \downarrow m-1} v_{-}^{\prime}(s)=+\infty$. When $B_{m}<m, \lim _{s \uparrow m} v_{-}^{\prime}(s)<$ $\lim _{s \downarrow B_{m}} v_{-}^{\prime}(s)<\lim _{s \uparrow B_{m}} v_{-}^{\prime}(s)<\lim _{s \downarrow m-1} v_{-}^{\prime}(s)=+\infty$.

(ii) There exist $C_{-}>c_{-}>0$ such that $c_{+}(s+1)^{\alpha_{-}} \leq v_{-}(s)+1 \leq C_{+}(s+1)^{\alpha_{-}}$ for all $s \geq 0$. Furthermore, $\lim _{s \rightarrow+\infty} v_{-}(s) /(s+1)^{\alpha_{-}}=1$.

(iii) The minimum value of $g_{-}(s):=\left(v_{-}(s)+1\right) /(s+1)^{\delta_{-}}$in $[0,+\infty)$ is 1 . Furthermore, when $\delta_{-}=\alpha_{-}<1$, any nonnegative integer is the minimizer. When $\delta_{-}<\alpha_{-} \leq 1$, the unique minimizer is 0 .

4.5. Optimal solution. Now, we are ready to present the optimal solution to problem (4.2) and compute its optimal value, denoted as $U^{*}$. Recall that we assume $\alpha_{+} \leq \delta_{+}, \delta_{-} \leq \alpha_{-}$, and $\alpha_{+} \leq \alpha_{-}$. The gambler does not play in the casino if and only if the optimal solution to problem (4.2) is $\boldsymbol{x}^{*}=\boldsymbol{y}^{*}=0$, and the gambler chooses to play if and only if there exist $\boldsymbol{x}$ and $\boldsymbol{y}$ such that $U(\boldsymbol{x}, \boldsymbol{y})>0$.

Theorem 4.5. Assume $\alpha_{+} \leq \delta_{+}, \delta_{-} \leq \alpha_{-}$, and $\alpha_{+} \leq \alpha_{-}$. Define

$$
f\left(y, s_{+}, s_{-}\right):=\left(v_{+}\left(s_{+}\right)+1\right)\left(\frac{s_{-}+1}{s_{+}+1}\right)^{\delta_{+}} y^{\delta_{+}}-\lambda\left(v_{-}\left(s_{-}\right)+1\right) y^{\delta_{-}}, \quad y, s_{+}, s_{-} \geq 0 .
$$

Copyright $@$ by SIAM. Unauthorized reproduction of this article is prohibited. 
(i) Suppose $\alpha_{+}=\delta_{+}=\alpha_{-}=\delta_{-}=1$. If $\lambda \geq 1$, the optimal solution to (4.2) is $\boldsymbol{x}^{*}=\boldsymbol{y}^{*}=0$, i.e., the gambler does not play in the casino. If $\lambda<1$, then $U^{*}=\infty$.

(ii) Suppose $\alpha_{+}=\delta_{+}<1$. Then, $U^{*}=\infty$ and there exist $s_{+}>0, x_{1}>0, \boldsymbol{y}$, and $\boldsymbol{z}_{+}^{(n)}, n \geq 1$ satisfying $\sum_{i=1}^{\infty} z_{+, i}^{(n)}=s_{+}$such that $\left(\boldsymbol{x}^{(n)}, \boldsymbol{y}\right)$ with $\boldsymbol{x}^{(n)}:=$ $\left(x_{1}, x_{1} \boldsymbol{z}_{+}^{(n)}\right)$ is feasible to problem $(4.2)$ and $U\left(\boldsymbol{x}^{(n)}, \boldsymbol{y}\right)$ diverges to infinity as $n \rightarrow \infty$.

(iii) Suppose $\delta_{-} \leq \delta_{+}$and $\alpha_{+}<\delta_{+}$. Define

$$
\begin{aligned}
& M_{1}:=\sup _{s_{+} \geq 0, s_{-} \geq 0}\left[\frac{\left(v_{+}\left(s_{+}\right)+1\right)\left(s_{-}+1\right)^{\delta_{+}}}{\left(v_{-}\left(s_{-}\right)+1\right)\left(s_{+}+1\right)^{\delta_{-}}\left(s_{+}+s_{-}+2\right)^{\delta_{+}-\delta_{-}}}\right], \\
& M_{2}:=\limsup _{s_{+}+s_{-} \rightarrow+\infty}\left[\frac{\left(v_{+}\left(s_{+}\right)+1\right)\left(s_{-}+1\right)^{\delta_{+}}}{\left(v_{-}\left(s_{-}\right)+1\right)\left(s_{+}+1\right)^{\delta_{-}}\left(s_{+}+s_{-}+2\right)^{\delta_{+}-\delta_{-}}}\right] .
\end{aligned}
$$

Then, $0<M_{1}<+\infty$ and $0 \leq M_{2} \leq M_{1}$. Furthermore, $M_{2}>0$ if and only if $\alpha_{-}=\alpha_{+}$or $\alpha_{-}=\delta_{-}$and $\bar{M}_{2}=M_{1}$ if $\alpha_{-}=\delta_{-}$. When $\lambda \geq M_{1}$, the optimal solution to (4.2) is $\boldsymbol{x}^{*}=\boldsymbol{y}^{*}=0$, i.e., the gambler does not play in the casino, and when $\lambda<M_{1}$, the gambler plays in the casino. When $M_{2}<\lambda<M_{1}$, $U^{*}<\infty$ and the optimal solution exists and is given as $\boldsymbol{x}^{*}=\left(x_{1}^{*}, x_{1}^{*} \boldsymbol{z}_{+}^{*}\left(s_{+}^{*}\right)\right)$, $\boldsymbol{y}^{*}=\left(y_{1}^{*}, y_{1}^{*} \boldsymbol{z}_{-}^{*}\left(s_{-}^{*}\right)\right)$, where

$$
\begin{aligned}
& \left(s_{+}^{*}, s_{-}^{*}\right) \in \underset{s_{+} \geq 0, s_{-} \geq 0}{\operatorname{argmax}} f\left(\hat{y}\left(s_{+}, s_{-}\right), s_{+}, s_{-}\right), \hat{y}\left(s_{+}, s_{-}\right):=\frac{s_{+}+1}{s_{+}+s_{-}+2}, \\
& y_{1}^{*}=\hat{y}\left(s_{+}^{*}, s_{-}^{*}\right), \quad x_{1}^{*}=\frac{s_{-}^{*}+1}{s_{+}^{*}+1} y_{1}^{*} .
\end{aligned}
$$

When $\lambda<M_{2}$, the optimal solution does not exist, $U^{*}=+\infty$ if $\alpha_{+}=$ $\alpha_{-}>\delta_{-}$and $U^{*}<+\infty$ if $\alpha_{+}<\alpha_{-}=\delta_{-}$, and there exist $\left(s_{+}^{(n)}, s_{-}^{(n)}\right)$ with $\lim _{n \rightarrow \infty} s_{-}^{(n)}=+\infty$ such that the objective value of the pair $\boldsymbol{x}^{(n)}:=$ $\left(x_{1}^{(n)}, x_{1}^{(n)} \boldsymbol{z}_{+}^{*}\left(s_{+}^{(n)}\right)\right), \boldsymbol{y}^{(n)}:=\left(y_{1}^{(n)}, y_{1}^{(n)} \boldsymbol{z}_{-}^{*}\left(s_{-}^{(n)}\right)\right)$, where

$$
y_{1}^{(n)}:=\frac{s_{+}^{(n)}+1}{s_{+}^{(n)}+s_{-}^{(n)}+2}, \quad x_{1}^{(n)}=\frac{s_{-}^{(n)}+1}{s_{+}^{(n)}+1} y_{1}^{(n)}
$$

converges to $U^{*}$ as $n \rightarrow+\infty$. Moreover, $s_{+}^{(n)} / s_{-}^{(n)}$ 's converge to zero when $\alpha_{-}=\delta_{-}$and are bounded from zero and from infinity when $\alpha_{-}=\alpha_{+}>\delta_{-}$.

(iv) Suppose $\delta_{+}<\delta_{-} \leq 1$. Then, the gambler plays in the casino. When $\alpha_{-}>\delta_{-}, U^{*}<\infty$ and the optimal solution is $\boldsymbol{x}^{*}=\left(x_{1}^{*}, x_{1}^{*} z_{+}^{*}\left(s_{+}^{*}\right)\right), \boldsymbol{y}^{*}=$ $\left(y_{1}^{*}, y_{1}^{*} z_{-}^{*}\left(s_{-}^{*}\right)\right)$, where

$$
\begin{gathered}
\left(s_{+}^{*}, s_{-}^{*}\right) \in \underset{s_{+} \geq 0, s_{-} \geq 0}{\operatorname{argmax}} f\left(\bar{y}\left(s_{+}, s_{-}\right), s_{+}, s_{-}\right), y_{1}^{*}=\bar{y}\left(s_{+}^{*}, s_{-}^{*}\right), x_{1}^{*}=\frac{s_{-}^{*}+1}{s_{+}^{*}+1} y_{1}^{*}, \\
\bar{y}\left(s_{+}, s_{-}\right):=\min \left\{\left(\frac{\delta_{+}\left(v_{+}\left(s_{+}\right)+1\right)\left(s_{-}+1\right)^{\delta_{+}}}{\delta_{-} \lambda\left(v_{-}(s)+1\right)\left(s_{+}+1\right)^{\delta_{+}}}\right)^{\frac{1}{\delta_{-}^{-\delta_{+}}}}, \frac{s_{+}+1}{s_{+}+s_{-}+2}\right\} .
\end{gathered}
$$

Copyright (C) by SIAM. Unauthorized reproduction of this article is prohibited. 
Moreover, if $\lambda \geq M_{3}$, where

$$
M_{3}:=\frac{\delta_{+}}{\delta_{-}}\left(s_{1}+2\right)^{\delta_{-}-\delta_{+}}\left(s_{1}+1\right)^{1-\delta_{-}}, \quad s_{1}:=\sum_{n=1}^{\infty}\left((n+1)^{\alpha_{+}}-n^{\alpha_{+}}\right)^{\frac{1}{1-\delta_{+}}},
$$

then we can take $s_{-}^{*}=0$ and $s_{+}^{*}=s_{1}$. When $\alpha_{-}=\delta_{-}$and $\lambda>M_{4}$, where

$$
M_{4}:=\frac{\delta_{+}}{\delta_{-}}\left(s_{1}+1\right)^{1-\delta_{-}}<M_{3},
$$

the optimal solution exists and is given as (4.19) with $s_{+}^{*}=s_{1}$ and $s_{-}=m$ for some nonnegative integer $m$. When $\alpha_{-}=\delta_{-}$and $\lambda \leq M_{4}, U^{*}<+\infty$, the optimal solution does not exist, and there exists $s_{+}^{*}$ such that the objective value of the pair $\boldsymbol{x}^{(n)}:=\left(x_{1}^{(n)}, x_{1}^{(n)} \boldsymbol{z}_{+}^{*}\left(s_{+}^{*}\right)\right), \boldsymbol{y}^{(n)}:=\left(y_{1}^{(n)}, y_{1}^{(n)} \boldsymbol{z}_{-}^{*}\left(s_{-}^{(n)}\right)\right)$ with $s_{-}^{(n)}=n, y_{1}^{(n)}:=\frac{s_{+}^{*}+1}{s_{+}^{*}+s_{-}^{(n)}+2}, x_{1}^{(n)}=\frac{s_{-}^{(n)}+1}{s_{+}^{*}+1} y_{1}^{(n)}$ converges to $U^{*}$ as $n \rightarrow+\infty$.

Except for the case $\delta_{+}>\alpha_{+}=\alpha_{-}>\delta_{-}, \lambda=M_{2}<M_{1}$ in which we do not know whether the optimal solution exists, we have solved problem (4.2) completely. When the optimal solution exists, it is provided in a closed form. Otherwise, a sequence of asymptotically optimal solutions are provided.

In the case $\alpha_{+}=\delta_{+}=\alpha_{-}=\delta_{-}=1$, the gambler does not play in the casino (i.e., the optimal strategy is $\boldsymbol{x}^{*}=\boldsymbol{y}^{*}=0$ ) when $\lambda \geq 1$ and chooses to play (because the optimal value is infinite and thus larger than the value of not gambling) when $\lambda<1$.

In the case $\alpha_{+}=\delta_{+}<1$, the optimal value is infinite. We find a sequence of asymptotically optimal solutions $\left(\boldsymbol{x}^{(n)}, \boldsymbol{y}\right)$. Note that the conditional expected gain is fixed to be $s_{+}+1$ and the conditional expected loss is also fixed. Thus, an infinite CPT value is achieved by taking strategies that have fixed and finite conditional expected gain and loss.

In the case $\delta_{-} \leq \delta_{+}$and $\alpha_{+}<\delta_{+}$, whether the optimal solution exists and whether the gambler plays in the casino depend on $\lambda$. When $\lambda \geq M_{1}$, the gambler chooses not to play; otherwise she plays. When $M_{2}<\lambda<M_{1}$, the optimal solution exists. Finally, when $\lambda<M_{2}$, which can happen only when $\alpha_{-}=\alpha_{+}$or when $\alpha_{-}=\delta_{-}$, the optimal solution does not exist. In this case, a sequence of asymptotically optimal solutions are given with $s_{-}^{(n)}$ going to infinity and $s_{+}^{(n)} / s_{-}^{(n)}$ converging to zero when $\alpha_{-}=\delta_{-}$or bounded from zero and from infinity when $\alpha_{-}=\alpha_{+}>\delta_{-}$. Recall that $s_{+}^{(n)}+1$ and $s_{-}^{(n)}+1$ stand for the conditional gain and loss, respectively. Therefore, when $\lambda<M_{2}$, the gambler takes a gain-exit strategy if $\alpha_{-}=\delta_{-}$and takes a nonexit strategy if $\alpha_{-}=\alpha_{+}>\delta_{-}$.

In the case $\delta_{+}<\delta_{-} \leq 1$, the gambler plays in the casino. When $\alpha_{-}>\delta_{-}$, the optimal solution exists, regardless of the value of $\lambda$. When $\alpha_{-}=\delta_{-}$, the optimal solution exists if and only if $\lambda>M_{4}$. When the optimal solution does not exist, a sequence of asymptotically optimal solutions are given with $s_{+}^{(n)}$ to be fixed and $s_{-}^{(n)}$ going to infinity. Therefore, the gambler takes a gain-exit strategy.

Finally, the optimal solution, if it exists, must take the form $\boldsymbol{x}^{*}=\left(x_{1}^{*}, x_{1}^{*} \boldsymbol{z}_{+}^{*}\left(s_{+}^{*}\right)\right)$, $\boldsymbol{y}^{*}=\left(y_{1}^{*}, y_{1}^{*} \boldsymbol{z}_{-}^{*}\left(s_{-}^{*}\right)\right)$ for some $x_{1}^{*} \geq 0, y_{1}^{*} \geq 0$, and $s_{ \pm}^{*} \geq 0$. Moreover, $s_{ \pm}^{*}$ can be solved by a two-dimensional optimization problem (e.g., (4.17) and (4.19)). Recall $\boldsymbol{z}_{+}^{*}(s)$ as defined in (4.6) and (4.8). When $\delta_{+}=1, z_{+, n}^{*}(s)=0$ for $n \geq\lfloor s\rfloor+2$, showing that the gambler plans to stop when reaching certain level of gains. When $\delta_{+}<1, z_{+, n}^{*}(s)>0$ for any $n \geq 1$, showing that the gambler chooses to continue with 
TABLE 2

Optimal solution to problem (4.2) when the utility function is piecewise power and the probability weighting functions are power. The third column shows whether the gambler enters the casino to gamble. The fourth column shows whether the optimal solution to problem (4.2) exists. The last column gives the optimal solution when it exists and the strategies (such as gain-exit, loss-exit, and nonexit strategies) the gambler takes when it does not. For the optimal solution, only $s_{+}^{*}, s_{-}^{*}$, and $y_{1}^{*}$ are listed. The optimal solution is then $\boldsymbol{x}^{*}=\left(x_{1}^{*}, x_{1}^{*} \cdot \boldsymbol{z}_{+}^{*}\left(s_{+}^{*}\right)\right), \boldsymbol{y}^{*}=\left(y_{1}^{*}, y_{1}^{*} \cdot \boldsymbol{z}_{-}^{*}\left(s_{-}^{*}\right)\right)$, where $x_{1}^{*}=\left(s_{-}^{*}+1\right) /\left(s_{+}^{*}+1\right) y_{1}^{*}$ and $\boldsymbol{z}_{+}^{*}(s)$ and $\boldsymbol{z}_{-}^{*}(s)$ are given as in (4.6), (4.8), (4.11), and (4.13), respectively. The functions $f, \hat{y}$, and $\bar{y}$ are given as in (4.15), (4.17), and (4.19), respectively. The blank cells indicate the only case in which problem (4.2) is not fully solved.

\begin{tabular}{|c|c|c|c|c|}
\hline & & Gam. & Exist. & Strategies \\
\hline \multirow{2}{*}{$\begin{array}{l}\alpha_{+}=\alpha_{-}= \\
\delta_{+}=\delta_{-}=1\end{array}$} & $\lambda \geq 1$ & No & Yes & not gamble \\
\hline & $\lambda<1$ & Yes & No & nonexit \\
\hline$\alpha_{+}>\delta_{+}$ & any $\lambda$ & Yes & No & loss-exit \\
\hline$\alpha_{-}<\delta_{-}$ & any $\lambda$ & Yes & No & gain-exit \\
\hline$\alpha_{-}<\alpha_{+}$ & any $\lambda$ & Yes & No & nonexit \\
\hline$\alpha_{+}=\delta_{+}<1$ & any $\lambda$ & Yes & No & certain asymptotical strategy \\
\hline \multirow{2}{*}{$\begin{array}{c}\delta_{-} \leq \delta_{+}, \\
\alpha_{+}<\delta_{+}, \text {and } \\
\alpha_{-}>\max \left(\alpha_{+}, \delta_{-}\right)\end{array}$} & $\lambda \geq M_{1}$ & No & Yes & not gamble \\
\hline & $\lambda<M_{1}$ & Yes & Yes & $\begin{array}{c}\left(s_{+}^{*}, s_{-}^{*}\right) \in \underset{1}{\operatorname{argmax}} f\left(\hat{y}\left(s_{+}, s_{-}\right), s_{+}, s_{-}\right) \\
y_{1}^{*}=\hat{y}\left(s_{+}^{*}, s_{-}^{*}\right)\end{array}$ \\
\hline \multirow{2}{*}{$\begin{array}{c}\delta_{-} \leq \delta_{+} \\
\alpha_{+}<\delta_{+}, \text {and } \\
\alpha_{-}=\delta_{-} \geq \alpha_{+}\end{array}$} & $\lambda \geq M_{1}$ & No & Yes & not gamble \\
\hline & $\lambda<M_{1}$ & Yes & No & gain-exit \\
\hline \multirow{4}{*}{$\begin{array}{c}\delta_{-} \leq \delta_{+}, \\
\alpha_{+}<\delta_{+}, \text {and } \\
\alpha_{-}=\alpha_{+}>\delta_{-}\end{array}$} & $\lambda \geq M_{1}$ & No & Yes & not gamble \\
\hline & $M_{2}<\lambda<M_{1}$ & Yes & Yes & $\begin{array}{c}\left(s_{+}^{*}, s_{-}^{*}\right) \in \underset{1}{\operatorname{argmax}} f\left(\hat{y}\left(s_{+}, s_{-}\right), s_{+}, s_{-}\right) \\
y_{1}^{*}=\hat{y}\left(s_{+}^{*}, s_{-}^{*}\right)\end{array}$ \\
\hline & $\lambda=M_{2}<M_{1}$ & Yes & & \\
\hline & $\lambda<M_{2}$ & Yes & No & nonexit \\
\hline \multirow{2}{*}{$\begin{array}{c}\alpha_{-}>\delta_{-}> \\
\delta_{+}>\alpha_{+}\end{array}$} & $\lambda \geq M_{3}$ & Yes & Yes & $s_{+}^{*}=s_{1}, s_{-}^{*}=0$, and $y_{1}^{*}=\bar{y}\left(s_{+}^{*}, s_{-}^{*}\right)$ \\
\hline & $\lambda<M_{3}$ & Yes & Yes & $\begin{array}{c}\left(s_{+}^{*}, s_{-}^{*}\right) \in \operatorname{argmax} f\left(\bar{y}\left(s_{+}, s_{-}\right), s_{+}, s_{-}\right) \\
\text {and } y_{1}^{*}=\bar{y}\left(s_{+}^{*}, s_{-}^{*}\right)\end{array}$ \\
\hline \multirow[t]{2}{*}{$\begin{array}{c}\alpha_{-}=\delta_{-}> \\
\delta_{+}>\alpha_{+}\end{array}$} & $\lambda>M_{4}$ & Yes & Yes & $\begin{array}{c}s_{+}^{*}=s_{1}, s_{-}^{*}=m \text { for some integer } m \\
\text { and } y_{1}^{*}=\bar{y}\left(s_{+}^{*}, s_{-}^{*}\right)\end{array}$ \\
\hline & $\lambda \leq M_{4}$ & Yes & No & gain-exit \\
\hline
\end{tabular}

positive probabilities at any level of gains. The gambler eventually stops at a certain level of gains because there exists a uniformly integrable and thus almost-surely-finite stopping time $\tau$ such that $S_{\tau}$ follows the optimal distribution. On the other hand, recall $\boldsymbol{z}_{-}^{*}(s)$ as defined in (4.11) and (4.13). We can see that $z_{-, n}^{*}(s)=0$ for $n \geq\lceil s\rceil+1$, showing that the gambler plans to stop with probability one when reaching a certain level of losses. Furthermore, in the optimal solution $\boldsymbol{y}^{*}=\left(y_{1}^{*}, y_{1}^{*} \boldsymbol{z}_{-}^{*}\left(s_{-}^{*}\right)\right)$, because $\boldsymbol{y}^{*}$ is the cumulative distribution of the optimal strategy, the gambler chooses to stop at no more than two loss levels (levels $\left\lceil s_{-}^{*}\right\rceil$ and $\left\lceil s_{-}^{*}\right\rceil+1$ when $\boldsymbol{z}_{-}^{*}\left(s_{-}^{*}\right)$ is in the form (4.11) and levels 1 and $\left\lceil s_{-}^{*}\right\rceil+1$ when $\boldsymbol{z}_{-}^{*}\left(s_{-}^{*}\right)$ is in the form (4.13)).

To summarize, if the optimal solution exists, the gambler stops as long as a random level of gains or a random level of losses is reached. The random level of losses can take no more than two values, so the gambler's loss is limited. When $\delta_{+}<1$ (which implies that the gambler overweighs large gains of small probabilities), the support of the random level of gains is the whole positive integer set, so the gambler effectively constructs a positively skewed payoff. A detailed summary of the results in Theorems 3.1 and 4.5 regarding the solution to problem (4.2) is given in Table 2.

We consider a numerical example to illustrate the optimal solution $\left(\boldsymbol{x}^{*}, \boldsymbol{y}^{*}\right)$ to (4.2) and the corresponding optimal strategy $\tau^{*}$ of the precommitted gambler. Denote 

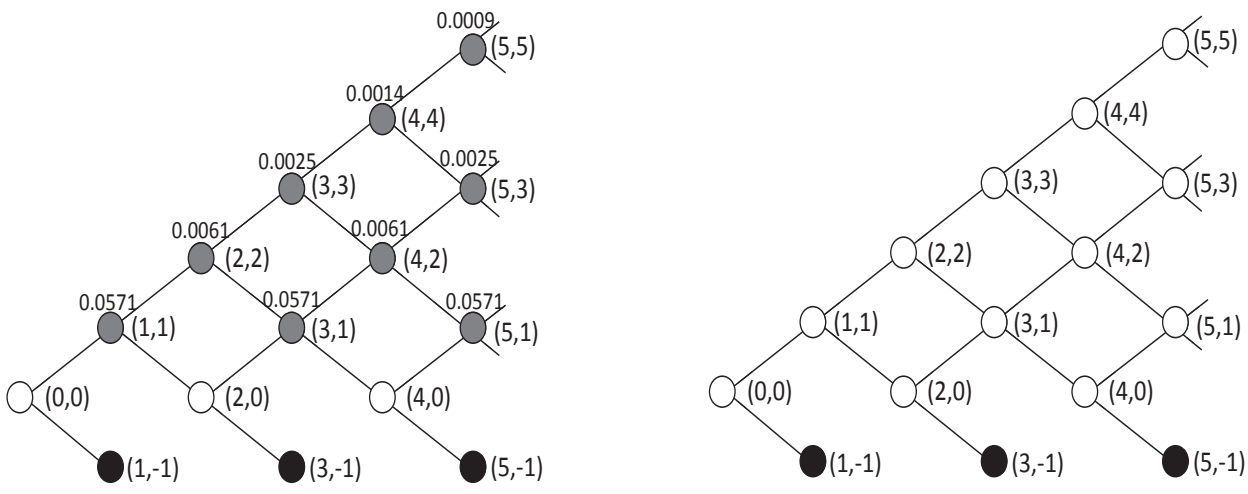

FIG. 2. Randomized path-independent stopping time (left panel) and randomized Azéma-Yor stopping (right panel) embedding the optimal probability measure in Example 1. Black nodes stand for "stop," white nodes stand for "continue," and gray nodes stand for the cases in which a random coin is tossed and the gambler stops if and only if the coin turns up tails. Each node is marked on the right by a pair $(t, x)$ representing time $t$ and $S_{t}=x$. Each gray node is marked on the top by a number showing the probability that the random coin tossed at that node turns up tails.

by $\left\{p_{n}^{*}\right\}$ the optimal distribution, i.e., the distribution of $S_{\tau^{*}}$. Obviously, $p_{n}^{*}=x_{n}^{*}-$ $x_{n+1}^{*}, n \geq 1, p_{-n}^{*}=y_{n}^{*}-y_{n+1}^{*}, n \geq 1$.

Example 1. We set $\alpha_{+}=0.5, \alpha_{-}=0.9, \delta_{ \pm}=0.52$, and $\lambda=2.25$. According to Theorem 4.5, the optimal solution to (4.2) exists: the optimal distribution is

$$
\begin{aligned}
& p_{n}^{*}=0.1360\left(\left(n^{0.5}-(n-1)^{0.5}\right)^{1 / 0.48}-\left((n+1)^{0.5}-n^{0.5}\right)^{1 / 0.48}\right), n \geq 2, \\
& p_{1}^{*}=0.0933, \quad p_{0}^{*}=0, \quad p_{-1}^{*}=0.8851, \quad p_{n}^{*}=0, n \leq-2 .
\end{aligned}
$$

The optimal distribution can be embedded into the symmetric random walk both by a randomized path-independent stopping time and by a randomized Azéma-Yor stopping time, which thus provide examples of feasible optimal strategies for the precommitted gambler. The construction of the stopping times can be found in [17], and here we only illustrate the stopping times in the first five periods in Figure 2 (the left panel for the randomized path-independent stopping time and the right panel for the randomized Azéma-Yor stopping time): the gain and loss of the gambler is represented by a recombining binomial tree. Black nodes stand for "stop," white nodes stand for "continue," and gray nodes stand for the cases in which a random coin is tossed and the gambler stops if and only if the coin turns up tails. Each node is marked on the right by a pair $(t, x)$ representing time $t$ and $S_{t}=x$. Each gray node is marked on the top by a number showing the probability that the random coin tossed at that node turns up tails. In both strategies, the gambler stops with probability one when the loss accumulates to 1 . On the other hand, in the randomized path-independent strategy, the gambler tosses a coin at each time to decide whether to stop, but in the randomized Azéma-Yor strategy, no coin is tossed in the first five periods. ${ }^{6}$ See more discussion on the difference between these two types of randomized strategies in [17].

\footnotetext{
${ }^{6}$ Indeed, we calculate that in the randomized Azéma-Yor strategy, the first time that the gambler may possibly stop at a level other than -1 is the first time his gain reaches 7 and comes back to 1 .
} 
5. Naive gamblers. Barberis [5] finds in his five-period casino gambling model that, due to the probability weighting, if the gambler revisits the gambling problem with the same CPT preferences in the future, she may find the initial strategy decided at time 0 to be no longer optimal. As a result, if she is unaware of this timeinconsistency, she may change to a new strategy that can be totally different from the initial one. Such gamblers are called naive gamblers.

Formally, suppose now that at time $T$, having played $T$ rounds, the gambler has arrived at some node and has not stopped yet with accumulated gain/loss equal to $H$. A naive gambler will reconsider the choice between continuing and leaving at that node. Following [5], we assume the reference point in the CPT preference of the gambler at any time to be the initial wealth level. As a result, the cumulative gain or loss of the gambler at any time $n \geq T$ is $S_{n}$. Conditioning on $S_{T}=H$, the gambling problem faced by the naive gambler is

$$
\max _{\tau \in \mathcal{T}_{T, H}} V_{T, H}\left(S_{\tau}\right),
$$

where $\mathcal{T}_{T, H}$ is the set of uniformly integrable randomized stopping times $\tau \geq T$ and

$$
\begin{aligned}
V_{T, H}\left(S_{\tau}\right)= & \sum_{n=1}^{\infty} u_{+}(n)\left(w_{+}\left(\mathbb{P}\left(S_{\tau} \geq n \mid S_{T}=H\right)\right)-w_{+}\left(\mathbb{P}\left(S_{\tau}>n \mid S_{T}=H\right)\right)\right) \\
& -\sum_{n=1}^{\infty} u_{-}(n)\left(w_{-}\left(\mathbb{P}\left(S_{\tau} \leq-n \mid S_{T}=H\right)\right)-w_{-}\left(\mathbb{P}\left(S_{\tau}<-n \mid S_{T}=H\right)\right)\right),
\end{aligned}
$$

where, as before, $V_{T, H}\left(S_{\tau}\right):=-\infty$ if the second sum of infinite series is infinite. One can see that the CPT value of the strategy of leaving the casino at $T$ is $u(H):=$ $u_{+}(H) \mathbf{1}_{H \geq 0}-u_{-}(H) \mathbf{1}_{H<0}$. As a result, the naive gambler decides to play at time $T$ with cumulative gain/loss level $H$ if and only if the optimal value of problem (5.1) is strictly larger than $u(H)$. Mathematically, (5.1) can be solved using exactly the same method as for solving problem (2.2) above.

The following corollary is parallel to Theorem 3.1, and the proof is omitted. For two integers $b<0<a$, we denote the relative first exit time after $T$ by

$$
\tau_{a, b}^{T}:=\inf \left\{n \geq T: S_{n}-S_{T}=a \text { or } S_{n}-S_{T}=b\right\} .
$$

Corollary 5.1. Fix time $T>0$ and level $H,-T \leq H \leq T$. Let $V_{T, H}^{*}$ denote the value of the optimization problem (5.1).

(i) Suppose (3.1) holds. Then, $V_{T, H}^{*}=\infty$ and there exists $b<0$ such that $\lim _{a \rightarrow+\infty} V_{T, H}\left(S_{\tau_{a, b}^{T}}\right)=+\infty$.

(ii) Suppose (3.2) holds. Then, $V_{T, H}^{*}=\sup _{x \geq 0} u_{+}(x)$. Moreover, the optimal solution to problem (5.1) does not exist and $V_{T, H}\left(S_{\tau_{a, b}^{T}}\right)$ with $a=\left\lfloor k^{\epsilon /(1+\epsilon)}\right\rfloor$ and $b=-k$ converges to $V_{T, H}^{*}$ as $k$ goes to infinity.

(iii) Assume $\lim _{x \rightarrow+\infty} u_{+}(x)=+\infty$ and suppose condition (3.3) holds. Then, $V_{T, H}^{*}=\infty$ and there exists $c>0$ such that $\lim _{k \rightarrow+\infty} V_{T, H}\left(S_{\tau_{a, b}^{T}}\right)=+\infty$, where $a=\lceil c k\rceil$ and $b=-k$.

Corollary 5.1 shows that when one of the conditions (3.1)-(3.3) is satisfied, the naive gambler, who adjusts her optimal strategy after each bet, always finds continuing gambling more attractive. In our general setting, this means that, at any time and for any level of gains or losses, with a positive probability she will continue to gamble. Further, since Corollary 5.1 only makes use of the simplest two-level exit strategies, it 
would also apply to a gambler who is restricted to only using nonrandomized and pathindependent strategies. Such a naive gambler would continue playing with probability one regardless of her gains or losses at any point in time.

With piecewise power utility function (3.4) and weighting functions (3.4), or weighting functions (3.5), or power weighting functions $w_{ \pm}(p)=p^{\delta_{ \pm}}$, the naive gambler never stops gambling with probability one if one of the conditions in Table 1 is satisfied. For weighting functions (3.6), the naive gambler never stops gambling with probability one if $\delta_{+}<1$ or if $\delta_{+}=1$ and $\alpha_{+}>a_{+}$.

Ebert and Strack [11] also study the conditions under which a naive gambler does not stop gambling. Our work differs from theirs in three respects. First, they assume that the naive gambler can construct strategies with arbitrarily small random payoffs and then show that the naive gambler prefers skewness in the small and thus does not stop gambling. This argument, however, does not work in our model because the stake size in the model is fixed at $\$ 1$. In fact, we show here that the naive gambler prefers skewness in the large. Indeed, in Corollary 5.1, for stopping time $\tau_{a, b}^{T}$ we send either $a$, or $b$, or both to infinity, and thus the payoff of $\tau_{a, b}^{T}$ can be very large. ${ }^{7}$ Second, Ebert and Strack [11] either assume that the utility function has finite left- and right-derivatives at 0 or consider the piecewise utility function as in (3.4) with $\alpha_{+}=\alpha_{-}$. We, however, also consider the case $\alpha_{+} \neq \alpha_{-}$. Third, we allow for randomized strategies while Ebert and Strack [11] use pure (i.e., nonrandomized) strategies only. Therefore, in their setting once they have proved that it is not optimal to stop, the immediate conclusion is that it is optimal to continue. In our setting, however, if it is not optimal to stop, we can only conclude that it is optimal to continue playing with a positive probability (i.e., the negation of "to stop with probability one" is "to continue with a positive probability") because we allow randomization.

When the optimal strategy of the precommitted gambler does not exist, which is the case when one of the conditions (3.1)-(3.3) holds, we cannot compare the optimal strategy of the precommitted gambler and the actual behavior of the naive gambler. Thus, in the following, we consider the case in which the optimal strategy of the precommitted gambler exists, which has already been solved in Theorem 4.5, and study the actual behavior of the naive gambler.

THEOREM 5.2.

(i) Assume $\delta_{+}<1$. For any $H \geq 1$, there exists feasible $(\boldsymbol{x}, \boldsymbol{y})$ to problem (5.1) such that $U(\boldsymbol{x}, \boldsymbol{y})>H^{\alpha_{+}}$.

(ii) Assume $\delta_{+}<\delta_{-}$. Then, for any $H \leq-1$, there exists feasible $(\boldsymbol{x}, \boldsymbol{y})$ to problem (5.1) such that $U(\boldsymbol{x}, \boldsymbol{y})>-\lambda(-H)^{\alpha_{-}}$.

Theorem 5.2(i) shows that if $\delta_{+}<1$, the naive gambler continues with a positive probability at any gain level, which is the same as a precommitted gambler. On the other hand, Theorem 5.2(ii) indicates that if $\delta_{+}<\delta_{-}$, which is consistent with most experimental estimates of CPT parameters in the literature (see Table 1), at each time, with a positive probability the naive gambler does not stop. More precisely, either she simply continues, or else she might want to toss a coin to decide whether to continue to play or not. In particular, if $\alpha_{+}<\delta_{+}<\delta_{-}<\alpha_{-}$, according to Theorem 4.5, the precommitted gambler stops with probability one if the loss hits a certain level. In sharp contrast, the naive gambler's actual behavior is to play with a positive probability at any loss level. Our finding of the naive gambler's behavior is

\footnotetext{
${ }^{7}$ In $[11$, Appendix W2], the authors also consider a preference for skewness in the large when the utility function is given by (3.4) with $\alpha_{+}=\alpha_{-}$.
}

Copyright (C) by SIAM. Unauthorized reproduction of this article is prohibited. 
TABLE 3

Probability mass function $p_{H, n}^{*}, n \in \mathbb{Z}$, of $S_{\tau_{H}^{*}}$ for the optimal solution $\tau_{H}^{*}$ to (5.1), $H=$ $\pm 1, \pm 2, \ldots, \pm 5$. Denote $q_{n}=\left(n^{0.5}-(n-1)^{0.5}\right)^{1 / 0.48}-\left((n+1)^{0.5}-n^{0.5}\right)^{1 / 0.48}, n \geq 1$.

\begin{tabular}{c|ccccccccccc}
\hline$H$ & $n \leq-7$ & -6 & -5 & -4 & -3 & -2 & -1 & 0 & 1 & 2 & $n \geq 3$ \\
\hline-5 & 0 & 0.98 & 0 & 0 & 0 & 0 & 0 & 0 & 0 & 0.0096 & $0.148 q_{n}$ \\
-4 & 0 & 0 & 0.97 & 0 & 0 & 0 & 0 & 0 & 0.0063 & $0.145 q_{n}$ & $0.145 q_{n}$ \\
-3 & 0 & 0 & 0 & 0.96 & 0 & 0 & 0 & 0 & 0.0161 & $0.142 q_{n}$ & $0.142 q_{n}$ \\
-2 & 0 & 0 & 0 & 0 & 0.95 & 0 & 0 & 0 & 0.0308 & $0.139 q_{n}$ & $0.139 q_{n}$ \\
-1 & 0 & 0 & 0 & 0 & 0 & 0.92 & 0 & 0 & 0.0543 & $0.136 q_{n}$ & $0.136 q_{n}$ \\
0 & 0 & 0 & 0 & 0 & 0 & 0 & 0.89 & 0 & 0.0933 & $0.136 q_{n}$ & $0.136 q_{n}$ \\
1 & 0 & 0 & 0 & 0 & 0 & 0 & 0 & 0.85 & $0.150 q_{n}$ & $0.150 q_{n}$ & $0.150 q_{n}$ \\
2 & 0 & 0 & 0 & 0 & 0 & 0 & 0 & 0.70 & $0.300 q_{n}$ & $0.300 q_{n}$ & $0.300 q_{n}$ \\
3 & 0 & 0 & 0 & 0 & 0 & 0 & 0 & 0.55 & $0.450 q_{n}$ & $0.450 q_{n}$ & $0.450 q_{n}$ \\
4 & 0 & 0 & 0 & 0 & 0 & 0 & 0 & 0.40 & $0.601 q_{n}$ & $0.601 q_{n}$ & $0.601 q_{n}$ \\
5 & 0 & 0 & 0 & 0 & 0 & 0 & 0 & 0.25 & $0.751 q_{n}$ & $0.751 q_{n}$ & $0.751 q_{n}$ \\
\hline
\end{tabular}

consistent with that in [11], but we allow for $\alpha_{+} \neq \alpha_{-}$. The explanation for the above stark difference between the behavior of the precommitted and the naive gamblers follows the logic outlined in [5] and recalled above in the introduction: at time 0 , the precommitted gambler decides to stop when the loss reaches a certain level, e.g., $L$, in the future because the probability of having losses strictly larger than $L$ is small from time 0's perspective and this small probability is exaggerated due to probability weighting. For the naive gambler, when she actually reaches loss level $L$ at some time $t$, the probability of having losses strictly larger than $L$ is no longer small from time $t$ 's perspective. Consequently, loss $L$ is not overweighted by the naive gambler at time $t$, so she chooses to take a chance and not to stop gambling.

Finally, we provide a numerical example to illustrate the actual behavior of the naive gambler.

Example 2. We use the same parameter values as in Example 1. We compute the optimal solution $\tau_{H}^{*}$ to (5.1) and the corresponding probability mass function of $S_{\tau_{H}^{*}}$, denoted as $p_{H, n}^{*}, n \in \mathbb{Z}$, for $H= \pm 1, \pm 2, \ldots, \pm 5$ to illustrate the actual behavior of the naive gambler. The details of how to find the solution are provided in the online supplementary materials (available at SSRN.2682637). The values of $p_{H, n}^{*}$ are shown in Table 3, where $q_{n}:=\left(n^{0.5}-(n-1)^{0.5}\right)^{1 / 0.48}-\left((n+1)^{0.5}-n^{0.5}\right)^{1 / 0.48}, n \geq 1$.

We can see that for $H \leq-1, p_{H, H}^{*}=0$, indicating that the naive gambler will play with probability one at any loss level. This strategy is in contrast to the optimal strategy of the precommitted gambler shown in Example 1: stop once the loss accumulates to $\$ 1$. On the other hand, for $H \geq 1, p_{H, H}^{*} \in(0,1)$, indicating that the naive gambler will play with some probability at any gain level.

We remark that the discussion in this section has been based on the assumption that the gambler does not change the reference point, an assumption also used in [5]. An alternative model is one in which the gambler adjusts the reference point endogenously for prior gains and losses. This is then likely to depend on the history of his winnings and promises to be a entirely different and difficult problem, which is left for future study.

6. Conclusion. In this paper, we generalized the casino model first proposed by [5] to an infinite-time setting and studied the optimal strategies of a precommitted gambler and a naive gambler. We found conditions which distinguish three important classes of optimal behavior of the precommitted gambler: loss-exit strategies, gainexit strategies, and nonexit strategies. Our theoretical results are consistent with 
the numerical results in [5]. When the utility function is piecewise power and the probability weighting functions are power, we derived the optimal strategy of the precommitted gambler analytically by solving an infinite-dimensional program. We found that the optimal strategy of the precommitted gambler, when it exists, is to cap the loss, i.e., to stop before the loss hits a certain level.

In the second part of the paper we studied the behavior of the naive gambler. We found conditions under which the optimal strategy of the precommitted gambler and the actual strategy implemented by the naive gambler are in stark contrast: the precommitted gambler must stop if her cumulative loss reaches a certain level whereas the naive gambler continues to play with a positive probability at any loss level.

\section{Appendix A. Proofs.}

Proof of Theorem 3.1. We prove (i) first. The martingale property of $S$ easily implies that $\mathbb{P}\left(S_{\tau_{a, b}}=a\right)=-b /(a-b), \mathbb{P}\left(S_{\tau_{a, b}}=b\right)=a /(a-b)$, and $\mathbb{P}\left(S_{\tau_{a, b}}=n\right)=0$, $n \notin\{a, b\}$. Consequently,

$$
\begin{aligned}
V\left(S_{\tau_{a, b}}\right) & =u_{+}(a) w_{+}(-b /(a-b))-u_{-}(-b) w_{-}(a /(a-b)) \\
& =u_{+}(-b(x-1)) w_{+}(1 / x)-u_{-}(-b) w_{-}(1-1 / x),
\end{aligned}
$$

where $x:=(a-b) /(-b)$. Choose $b \in \mathbb{Z}$ such that $-b \geq 2 s$. Then, $-b(x-1) \geq s x$ for any $x \geq 2$. Consequently, as $a \rightarrow+\infty$, we have $x \rightarrow+\infty$ and

$$
V\left(S_{\tau_{a, b}}\right) \geq u_{+}(s x) w_{+}(1 / x)-u_{-}(-b) w_{-}(1-1 / x) \rightarrow+\infty .
$$

Next, we prove (ii). We have

$$
\begin{aligned}
V\left(S_{\tau_{a, b}}\right) & =u_{+}(a) w_{+}(-b /(a-b))-u_{-}(-b) w_{-}(a /(a-b)) \\
& =u_{+}\left(\left\lfloor k^{\epsilon /(1+\epsilon)}\right\rfloor\right) w_{+}(1-1 / y)-u_{-}(k) w_{-}(1 / y),
\end{aligned}
$$

where $y:=(a-b) / a$. We can see that

$$
y=1-b / a=1+k /\left\lfloor k^{\epsilon /(1+\epsilon)}\right\rfloor>k / k^{\epsilon /(1+\epsilon)}=k^{1 /(1+\epsilon)} .
$$

As a result,

$$
\limsup _{k \rightarrow+\infty} u_{-}(k) w_{-}(1 / y) \leq \limsup _{k \rightarrow+\infty} u_{-}(k) w_{-}\left(1 / k^{1 /(1+\epsilon)}\right)=0 .
$$

In addition, as $k \rightarrow+\infty$, we have $w_{+}(1-1 / y)$ goes to 1 . Consequently,

$$
\lim _{k \rightarrow+\infty} V\left(S_{\tau_{a, b}}\right)=\sup _{x>0} u_{+}(x) .
$$

On the other hand, it is straightforward to see that for any feasible $\tau, V\left(S_{\tau}\right)<$ $\sup _{x>0} u_{+}(x)$. Thus, problem $(2.2)$ is ill-posed and the optimal value is $\sup _{x>0} u_{+}(x)$.

Finally, we prove (iii). Suppose

$$
\limsup _{x \rightarrow+\infty} u_{-}\left(x p_{0} /\left(1-p_{0}\right)\right) / u_{+}(x)<w_{+}\left(p_{0}\right) / w_{-}\left(1-p_{0}\right)
$$

for some $p_{0} \in(0,1)$. Then, there exists $\delta_{0}>0$ and $\varepsilon_{0}>0$ such that

$$
\frac{w_{+}(p)}{w_{-}(1-p)}>\limsup _{x \rightarrow+\infty} \frac{u_{-}\left(x p_{0} /\left(1-p_{0}\right)\right)}{u_{+}(x)}+\varepsilon_{0} \quad \forall p \in\left[p_{0}-\delta_{0}, p_{0}+\delta_{0}\right] .
$$

Copyright (c) by SIAM. Unauthorized reproduction of this article is prohibited. 
Choose $c>0$ such that $1 /(1+c)=p_{0}$. Then, for sufficiently large $k$, we have $k /(\lceil c k\rceil+k) \in\left[p_{0}-\delta_{0}, p_{0}+\delta_{0}\right]$. Consequently, as $k$ goes to $+\infty$,

$$
\begin{aligned}
V\left(S_{\tau_{a, b}}\right) & =u_{+}(\lceil c k\rceil) w_{+}(k /(\lceil c k\rceil+k))-u_{-}(k) w_{-}(\lceil c k\rceil /(\lceil c k\rceil+k)) \\
& =u_{+}(\lceil c k\rceil) w_{-}(\lceil c k\rceil /(\lceil c k\rceil+k))\left[\frac{w_{+}(k /(\lceil c k\rceil+k))}{w_{-}(\lceil c k\rceil /(\lceil c k\rceil+k))}-\frac{u_{-}(k)}{u_{+}(\lceil c k\rceil)}\right] \\
& \geq u_{+}(\lceil c k\rceil) w_{-}\left(1-\left(p_{0}+\delta_{0}\right)\right)\left[\frac{w_{+}(k /(\lceil c k\rceil+k))}{w_{-}(\lceil c k\rceil /(\lceil c k\rceil+k))}-\frac{u_{-}(k)}{u_{+}(c k)}\right] \\
& =u_{+}(\lceil c k\rceil) w_{-}\left(1-\left(p_{0}+\delta_{0}\right)\right)\left[\frac{w_{+}(k /(\lceil c k\rceil+k))}{w_{-}(\lceil c k\rceil /(\lceil c k\rceil+k))}-\frac{u_{-}(k)}{u_{+}\left(k\left(1-p_{0}\right) / p_{0}\right)}\right] \\
& \geq u_{+}(\lceil c k\rceil) w_{-}\left(1-\left(p_{0}+\delta_{0}\right)\right) \varepsilon_{0} \rightarrow+\infty .
\end{aligned}
$$

Proof of Theorem 5.2. As in the case of problem (2.2), using the Skorohod embedding results in [17], we can show that problem (5.1) is equivalent to

$$
\begin{aligned}
& \operatorname{Max}_{\boldsymbol{x}, \boldsymbol{y}} U(\boldsymbol{x}, \boldsymbol{y}) \\
& \text { subject to } 1 \geq x_{1} \geq x_{2} \geq \cdots \geq x_{n} \geq \cdots \geq 0 \text {, } \\
& 1 \geq y_{1} \geq y_{2} \geq \cdots \geq y_{n} \geq \cdots \geq 0 \\
& x_{1}+y_{1} \leq 1, \sum_{n=1}^{\infty} x_{n}=\sum_{n=1}^{\infty} y_{n}+H \text {. }
\end{aligned}
$$

Using the same argument as for problem (4.2), we can show that problem (A.1) is equivalent to

$$
\begin{array}{cl}
\underset{x_{1}, y_{1}, s_{+}, s_{-}}{\operatorname{Max}} & \left(v_{+}\left(s_{+}\right)+1\right) x_{1}{ }^{\delta_{+}}-\lambda\left(v_{-}\left(s_{-}\right)+1\right) y_{1}{ }^{\delta_{-}} \\
\text {subject to } & x_{1}+y_{1} \leq 1,\left(s_{+}+1\right) x_{1}=\left(s_{-}+1\right) y_{1}+H, \\
& x_{1} \geq 0, y_{1} \geq 0, s_{+} \geq 0, s_{-} \geq 0
\end{array}
$$

Denote

$$
F_{H}\left(y_{1}, s_{+}, s_{-}\right):=\left(v_{+}\left(s_{+}\right)+1\right)\left(\frac{s_{-}+1}{s_{+}+1}\right)^{\delta_{+}}\left(y_{1}+\frac{H}{s_{-}+1}\right)^{\delta_{+}}-\lambda\left(v_{-}\left(s_{-}\right)+1\right) y_{1}{ }^{\delta_{-}} .
$$

Then, when $H \geq 1$, problem (A.2) is equivalent to

$$
\begin{array}{cl}
\underset{y_{1}, s_{+}, s_{1}}{\operatorname{Max}} & F_{H}\left(y_{1}, s_{+}, s_{-}\right) \\
\text {subject to } & 0 \leq y_{1} \leq \frac{s_{+}+1-H}{s_{+}+s_{-}+2}, s_{+} \geq H-1, s_{-} \geq 0 .
\end{array}
$$

When $H \leq-1$, problem (A.2) is equivalent to

$$
\begin{array}{cl}
\underset{y_{1}, s_{+}, s_{1}}{\max } & F_{H}\left(y_{1}, s_{+}, s_{-}\right) \\
\text {subject to } & \frac{-H}{s_{-}+1} \leq y_{1} \leq \frac{s_{+}+1-H}{s_{+}+s_{-}+2}, s_{+} \geq 0, s_{-} \geq-H-1 .
\end{array}
$$

We first assume $\delta_{+}<1$ and consider $H \geq 1$. For $H=1$, we choose $x_{1}=\frac{1}{s_{1}+1}$, $y_{1}=0, s_{+}=s_{1}, s_{-}=0$, which is a feasible solution to (A.2). Moreover, the objective value is $\left(v_{+}\left(s_{1}\right)+1\right) /\left(s_{1}+1\right)^{\delta_{+}}=\left(1+s_{1}\right)^{1-\delta_{+}}>1$, where the equality is the case because of Proposition 4.2(iii) and the inequality is the case because $\delta_{+}<1$. For $H \geq 2$, we have $F_{H}(0, H-1,0)=v_{+}(H-1)+1>H^{\alpha_{+}}$, where the inequality is the 
case because of Proposition 4.1(iv). Therefore, for any $H \geq 1$, we can find feasible $(\boldsymbol{x}, \boldsymbol{y})$ such that $U(\boldsymbol{x}, \boldsymbol{y})>H^{\alpha_{+}}$.

Next, we assume $\delta_{+}<\delta_{-}$and consider $H \leq-1$. Consider $s_{+}=0, y_{1}:=$ $(1-H) /\left(s_{-}+2\right)$, and the corresponding objective value

$$
h\left(s_{-}\right):=F_{H}\left(\frac{1-H}{s_{-}+2}, 0, s_{-}\right)=\left(\frac{s_{-}+H+1}{s_{-}+2}\right)^{\delta_{+}}-\lambda\left(v_{-}\left(s_{-}\right)+1\right)\left(\frac{1-H}{s_{-}+2}\right)^{\delta_{-}} .
$$

According to Proposition 4.3(ii), $v_{-}^{\prime}(s)=\delta_{-}\left((-H)^{\alpha_{-}}-(-H-1)^{\alpha_{-}}\right)(s+H+1)^{\delta_{-}-1}$ for $s$ in a neighborhood of $-H-1$. Consequently, we have

$$
\begin{aligned}
h^{\prime}\left(s_{-}\right)= & \delta_{+}\left(\frac{s_{-}+H+1}{s_{-}+2}\right)^{\delta_{+}-1} \frac{1-H}{\left(s_{-}+2\right)^{2}} \\
& +\delta_{-} \lambda\left(v_{-}\left(s_{-}\right)+1\right)\left(\frac{1-H}{s_{-}+2}\right)^{\delta_{-}-1} \frac{1-H}{\left(s_{-}+2\right)^{2}} \\
& -\lambda \delta_{-}\left((-H)^{\alpha_{-}}-(-H-1)^{\alpha_{-}}\right)\left(s_{-}+H+1\right)^{\delta_{-}-1}\left(\frac{1-H}{s_{-}+2}\right)^{\delta_{-}} \\
\geq & {\left[\delta_{+}\left(s_{-}+H+1\right)^{\delta_{+}-\delta_{-}} \frac{(1-H)^{1-\delta_{-}}}{\left(s_{-}+2\right)^{1+\delta_{+}-\delta_{-}}}-\lambda \delta_{-}\left((-H)^{\alpha_{-}}-(-H-1)^{\alpha_{-}}\right)\right] } \\
& \times\left(s_{-}+H+1\right)^{\delta_{-}-1}\left(\frac{1-H}{s_{-}+2}\right)^{\delta_{-}} .
\end{aligned}
$$

Because $\delta_{+}<\delta_{-}$, we conclude $\lim _{s_{-} \downarrow-H-1} h^{\prime}\left(s_{-}\right)>0$. From Proposition 4.3(ii), we have $v_{-}(-H-1)=(-H)^{\alpha_{-}}-1$, so $h(-H-1)=-\lambda(-H)^{\alpha_{-}}$. Therefore, there exists $s_{-}>-H-1$ such that $h\left(s_{-}\right)>-\lambda(-H)^{\alpha_{-}}$. Consequently, there exists feasible $(\boldsymbol{x}, \boldsymbol{y})$ to problem $\left(\right.$ A.1) such that $U(\boldsymbol{x}, \boldsymbol{y})>-\lambda(-H)^{-\alpha_{-}}$.

Acknowledgments. The authors thank three anonymous referees for their comments that have led to improvement of the paper. They are also grateful to comments by participants in the INFORMS 2016 Annual Meeting at Nashville and in the Symposium on Financial Risk Management: Topics on Time Consistency at The Chinese University of Hong Kong. A preliminary part of this research was completed while Jan Obłój was visiting CUHK in March 2013 and he is grateful for the support from the host.

\section{REFERENCES}

[1] M. Abdellaoui, Parameter-free elicitation of utility and probability weighting functions, Management Sci., 46 (2000), pp. 1497-1512.

[2] M. Agranov and P. Ortoleva, Stochastic choice and preferences for randomization, J. Polit. Economy, 125 (2017), pp. 40-68.

[3] E. B. Andrade And G. IYer, Planned versus actual betting in sequential gambles, J. Marketing Res., 46 (2009), pp. 372-383.

[4] E. M. Azevedo And D. Gottlieb, Risk-neutral firms can extract unbounded profits from consumers with prospect theory preferences, J. Econom. Theory, 147 (2012), pp. 12911299.

[5] N. Barberis, A model of casino gambling, Management Sci., 58 (2012), pp. 35-51.

[6] R. Barkan and J. R. Busemeyer, Changing plans: Dynamic inconsistency and the effect of experience on the reference point, Psychonomic Bull. Rev., 6 (1999), pp. 547-554.

[7] A. Boolu, B. van PraAg, and G. van de Kuilen, A parametric analysis of prospect theory's functionals for the general population, Theory and Decision, 68 (2010), pp. 115-148.

Copyright (c) by SIAM. Unauthorized reproduction of this article is prohibited. 
[8] C. F. Camerer And T.-H. Ho, Violations of the betweenness axiom and nonlinearity in probability, J. Risk Uncertainty, 8 (1994), pp. 167-196.

[9] A. M. Cox And J. OBŁóJ, Classes of measures which can be embedded in the simple symmetric random walk, Electron. J. Probab., 13 (2008), pp. 1203-1228.

[10] N. Dwenger, D. Kübler, and G. Weizsacker, Flipping a Coin: Theory and Evidence, SSRN:2353282, 2013.

[11] S. Ebert And P. StRAck, Until the bitter end: On prospect theory in a dynamic context, Amer. Econ. Rev., 105 (2015), pp. 1618-1633.

[12] S. Ebert and P. Strack, Never, Ever Getting Started: On Prospect Theory Without Commitment, SSRN:2765550, 2017.

[13] N. Etchart-Vincent, Is probability weighting sensitive to the magnitude of consequences? An experimental investigation on losses, J. Risk Uncertainty, 28 (2004), pp. 217-235.

[14] H. Fehr-Duda, M. De Gennaro, and R. Schubert, Gender, financial risk, and probability weights, Theory and Decision, 60 (2006), pp. 283-313.

[15] W. M. Goldstein And H. J. Einhorn, Expression theory and the preference reversal phenomena, Psychol. Rev., 94 (1987), pp. 236-254.

[16] X. D. He, S. Hu, J. ObєóJ, And X. Y. Zhou, Randomized and path-dependent strategies in Barberis' casino gambling model, Oper. Res., 65 (2017), pp. 97-103.

[17] X. D. He, S. Hu, J. ObєóJ, And X. Y. Zhou, Two explicit Skorokhod embeddings for simple symmetric random walk, Stochastic Process. Appl., 2018; also available online at arXiv:1705.05635.

[18] X. D. He AND X. Y. ZHou, Portfolio choice under cumulative prospect theory: An analytical treatment, Management Sci., 57 (2011), pp. 315-331.

[19] V. Henderson, D. Hobson, And A. TSE, Randomized strategies and prospect theory in a dynamic context, J. Econom. Theory, 168 (2017), pp. 287-300.

[20] V. Henderson, D. Hobson, And A. S. Tse, Probability Weighting, Stop-Loss and the Disposition Effect, SSRN:2823449, 2017.

[21] S. Hu, Optimal Exist Strategies of Behavioral Gamblers, Ph.D. thesis, The Chinese University of Hong Kong, 2014.

[22] Y.-J. Huang, A. NGuyen-HuU, And X. Y. Zhou, General stopping behaviors of naive and noncommitted sophisticated agents, with application to probability distortion, Math. Finance, to appear.

[23] A. Imas, The realization effect: Risk-taking after realized versus paper losses, Amer. Econ. Rev., 106 (2016), pp. 2086-2109.

[24] H. Jin AND X. Y. Zhou, Behavioral portfolio selection in continuous time, Math. Finance, 18 (2008), pp. 385-426

[25] H. Jin AND X. Y. Zhou, Greed, leverage, and potential losses: A prospect theory perspective, Math. Finance, 23 (2013), pp. 122-142.

[26] D. Kahneman, J. L. Knetsch, And R. H. Thaler, Experimental tests of the endowment effect and the Coase theorem, J. Polit. Economy, 98 (1990), pp. 1325-1348.

[27] D. Kahneman and A. Tversky, Prospect theory: An analysis of decision under risk, Econometrica, 47 (1979), pp. 263-291.

[28] V. Köbberling And P. P. Wakker, An index of loss aversion, J. Econom. Theory, 122 (2005), pp. 119-131.

[29] R. A. Pollak, Consistent planning, Rev. Econ. Stud., 35 (1968), pp. 201-208.

[30] D. Prelec, The probability weighting function, Econometrica, 66 (1998), pp. 497-527.

[31] J. Quiggin, A theory of anticipated utility, J. Econ. Behav. Organ., 3 (1982), pp. 323-343.

[32] R. H. Strotz, Myopia and inconsistency in dynamic utility maximization, Rev. Econ. Stud., 23 (1955-1956), pp. 165-180.

[33] A. TVersky and D. Kahneman, Advances in prospect theory: Cumulative representation of uncertainty, J. Risk Uncertainty, 5 (1992), pp. 297-323.

[34] P. Wakker and A. Tversky, An axiomatization of cumulative prospect theory, J. Risk Uncertainty, 7 (1993), pp. 147-175.

[35] G. Wu And R. Gonzalez, Curvature of the probability weighting function, Management Sci., 42 (1996), pp. $1676-1690$.

[36] Z. Q. Xu And X. Y. Zhou, Optimal stopping under probability distortion, Ann. Appl. Probab., 23 (2012), pp. 251-282.

Copyright (c) by SIAM. Unauthorized reproduction of this article is prohibited. 